\title{
Newcastle Disease Virus Expressing an Angiogenic Inhibitor Exerts an Enhanced Therapeutic Efficacy in Colon Cancer Model
}

\section{Fanrui Meng}

Northeast Agricultural University

\section{Yukai Cao}

Northeast Agricultural University

Han Su

Northeast Agricultural University

Limin Tian

Northeast Agricultural University

Jiarui Yang

Jiangsu Kanion Pharmaceutical CO. LTD, Jiangsu, Lianyungang. State Key Laboratory of New-tech for Chinese Medicine Pharmaceutical Process, Jiangsu, China

\section{Wei Xiao}

Jiangsu Kanion Pharmaceutical CO. LTD, Jiangsu, Lianyungang. State Key Laboratory of New-tech for Chinese Medicine Pharmaceutical Process, Jiangsu, China

Deshan Li ( $\square$ deshanli@163.com )

Northeast Agricultural University

\section{Research Article}

Keywords: rNDV, VEGF-Trap, Gene therapy, Anti-angiogenesis, Colon cancer

Posted Date: January 21st, 2021

DOl: https://doi.org/10.21203/rs.3.rs-149651/v1

License: (c) (i) This work is licensed under a Creative Commons Attribution 4.0 International License. Read Full License

Version of Record: A version of this preprint was published at PLOS ONE on April 5th, 2022. See the published version at https://doi.org/10.1371/journal.pone.0264896. 


\title{
Newcastle disease virus expressing an angiogenic inhibitor exerts an
}

\section{enhanced therapeutic efficacy in colon cancer model}

Fanrui Meng ${ }^{1}$, Yukai $\mathrm{Cao}^{1}$, Han $\mathrm{Su}^{1}$, Limin Tian ${ }^{1}$, Jiarui Yang ${ }^{2}$, Wei Xiao ${ }^{2 * *}$, Deshan $\mathrm{Li}^{1 *}$

${ }^{1}$ Northeast Agricultural University, Harbin, China

${ }^{2}$ Jiangsu Kanion Pharmaceutical CO. LTD, Jiangsu, Lianyungang. State Key Laboratory of New-tech for Chinese Medicine Pharmaceutical Process, Jiangsu, China

Fanrui Meng Northeast Agricultural University, Harbin, China 1192841993@qq.com Yukai Cao Northeast Agricultural University, Harbin, China 1179778594@qq.com Han Su Northeast Agricultural University, Harbin, China 727690458@qq.com Limin Tian Northeast Agricultural University, Harbin, China 2676690763@qq.com Jiarui Yang Jiangsu Kanion Pharmaceutical CO. LTD, Jiangsu, Lianyungang. State Key Laboratory of New-tech for Chinese Medicine Pharmaceutical Process, Jiangsu, China1260481892@qq.com

Wei Xiao Jiangsu Kanion Pharmaceutical CO. LTD, Jiangsu, Lianyungang. State Key Laboratory of New-tech for Chinese Medicine Pharmaceutical Process, Jiangsu, China Xw_kanion@163.com (second corresponding author)

Deshan Li Northeast Agricultural University, Harbin, China deshanli@163.com (first corresponding author)

*Corresponding authors at: School of Life Science, Northeast Agricultural University, Harbin, Heilongjiang Province, 150030, China, E-mail addresses: deshanli@163.com (DS, Li first corresponding author).

**Corresponding authors at: Jiangsu Kanion Pharmaceutical CO. LTD, Jiangsu, Lianyungang. State Key Laboratory of New-tech for Chinese Medicine Pharmaceutical Process, Jiangsu, 220000, China. E-mail addresses: Xw_kanion@163.com ( W, Xiao second corresponding author)

\begin{abstract}
Numerous studies demonstrate that the NDV-mediated gene therapy is a promising new approach for treatment of cancer. VEGF-Trap plays a vital role in anti-angiogenesis. Therefore, we hypothesize that a recombinant NDV (rNDV) expressing VEGF-Trap would be an ideal agent for the colon cancer therapy. In this study, VEGF-Trap gene was incorporated into the genome of rNDV (named rNDV-VEGF-Trap). rNDV-VEGF-Trap reduced cell growth ratio by $85.37 \%$ and migration ratio by $87.9 \%$ in EA.hy926 cells. In vivo studies, treatment with rNDV-VEGF-Trap reduced tumor volume of CT26-bearing mice by more than 3 folds and tumor weight by more than 4 folds. Immunohistochemistry analysis of CD34 showed rNDV-VEGF-Trap significantly decreased the number of vascular endothelial
\end{abstract}


cells in the tumor tissues of the tumor-bearing mice. Moreover, Western blot analysis demonstrated that treatment with rNDV-VEGF-Trap significantly decreased the phosphorylation levels of AKT, ERK1/2 and STAT3 and increased the expression levels of P53, BAX and cleaved caspase-3 in the tumor tissue. In addition, to evaluate the toxicity of VEGF-Trap, serum chemistries were analyzed. The results showed that rNDV-VEGF-Trap caused insignificant changes of creatinine levels, alanine aminotransferase (ALT) and aspartate transaminase (AST). Futhermore, administration of rNDV-VEGF-Trap did not cause the diarrhoea, decreased appetite, weight decrease and haemorrhage of the experimental mice. These data suggest that rNDV-VEGF-Trap exhibits an enhanced inhibition of CT26-bearing mice by enhancing anti-angiogenesis and apoptosis. rNDV-VEGF-Trap is a potential candidate for carcinoma therapy especially for colon cancer.

Keywords: rNDV, VEGF-Trap, Gene therapy, Anti-angiogenesis, Colon cancer

\section{Introduction}

As one of the main causes of human cancer death, the mortality and morbidity of colon cancer continued to rise ${ }^{1}$. Currently, surgery, radiotherapy and chemotherapy are the major strategies for colon cancer, but the high recurrence rate and adverse reactions decreased overall survival rate ${ }^{2}$. Colon cancer, which is similar to most other malignancies, has the risk of malignant invasion and metastasis ${ }^{3,4}$. One-half of patients with colon cancer will develop liver or lung metastasis, which are associated with abundant vascular distribution ${ }^{5}$. Therefore, optimizing the treatment strategy is a major challenge to improve the survival rate ${ }^{6-8}$. Besides traditional methods, immunotherapy has become a very hot field, which includes oncolytic viruses, tumor vaccines and immune checkpoint inhibitors etc ${ }^{9-14}$. As the development of genetic engineering and related disciplines, oncolytic viruses have been widely applied to treat colon cancer in recent years ${ }^{15}$.

Newcastle disease virus (NDV) is affiliated to the family Paramyxoviridae, which is an avian paramyxovirus type I virus ${ }^{16-17}$. The 15186 nucleotides of NDV genome encode six structural proteins, which are nucleocapsid protein (NP), phosphoprotein (P), RNA-dependent RNA polymerase (L), matrix protein (M), hemagglutinin-neuraminidase $(\mathrm{HN})$ and fusion protein $(\mathrm{F})^{18,19}$. NDV causes severe infection in avian species, but there is no significant symptoms to humans ${ }^{20}$. It exbits oncolytic ability through mainly replicates in tumor cells rather than normal cells ${ }^{21,22}$. The oncolytic potency relies on the ability to induce apoptosis in infected cancer cells ${ }^{23,24}$. However, it is not enough to treat tumor only depending on the oncolytic ability of $\mathrm{NDV}^{25,26}$.

Angiogenesis plays a crucial role in tumor growth and metastasis ${ }^{27,28}$. The growth, local tissue invasion and distant metastasis of colon cancer are highly dependent on angiogenesis ${ }^{29,30}$. Colon cancer is one of the ideal angiogenesis-dependent solid tumors. In tumor cells, the activation of oncogenes and inactivation of tumor suppressor genes lead to the upregulated expression of VEGF 31,32 . Overproduction of VEGF leads to abnormal changes in tumor vessels, such as distortion, fragmentation, and lack of pericytes. These dysfunctional and leaky vessels 
promote the development of tumor tissue microenvironment that limits the delivery of anti-tumor drugs to the targets ${ }^{33}$. VEGF and its signaling pathway represent attractive targets for the treatment of cancer, as this growth factor plays a role in various cancer types as an important pro-angiogenic cytokine ${ }^{34}$. A previous study describes anti-angiogenic therapy using vascular endothelial growth factor trap (named VEGF-Trap or aflibercept), which is an effective protein that blocks the tumor-associated VEGF signaling pathway. VEGF-Trap, which is a fusion protein, is composed of some extracellular domains of VEGFR-1 and VEGFR-2 and FC segment of human $\mathrm{IgG}$ antibody ${ }^{35}$. VEGF-Trap blocks the signal transduction of VEGF/VEGFR and inhibit angiogenesis and the tumor growth by binding with VEGF $^{36}$. However, repeated injections of VEGF-Trap lead to adverse reactions such as AST increase, serum creatinine increase, ALT increase, diarrhea, decreased appetite, weight decrease and haemorrhage of the patients ${ }^{37}$. Therefore, the treatment of VEGF-Trap still needs to overcome these limitations.

In the present study, we generated rNDV-VEGF-Trap and evaluated its anti-angiogenesis, antitumor efficacy and security in vitro and in vivo. These data suggest that the virus possesses a significant inhibiting effect against vascular endothelial cells line EA.hy926 and exhibits an effective anti-angiogenesis, oncolytic activity and security in the CT26 tumor mouse model.

\section{Materials and methods}

\section{Virus and Cell lines}

The strain E.coli DH5a, strain E.coli STBL2, rNDV vector, VEGF-Trap gene, auxiliary plasmid (PTM-NP, PTM-P and PTM-L), human colon cancer cell line (HCT116), human umbilical vein endothelial cell line (EA.hy926), mouse colon cancer cell line (CT26), mouse breast cancer cell line (4T-1), hamster kidney cell line (BHK-21), were supplied by northeast agricultural university biological pharmaceutical teaching and research section. CT26 and 4T1 were maintained in RPMI 1640 supplemented with 10\% (v/v) FBS, 1\% (v/v) penicillin/streptomycin. BHK-21 were maintained in DMEM containing 10\% new-born calf serum (NCS) and $1 \%$ penicillin/streptomycin. HCT116 was maintained in McCoy'5A supplemented with $10 \%(\mathrm{v} / \mathrm{v})$ FBS, $1 \%(\mathrm{v} / \mathrm{v})$ penicillin/streptomycin. All cell lines were authenticated using Short Tandem Repeat profiling, tested for mycoplasma contamination and grown at $37^{\circ} \mathrm{C}$ humidified incubator with $5 \% \mathrm{CO} 2$ atmosphere.

\section{Other reagents}

DMEM, McCoy'5A, RPMI 1640 were purchased from GIBCO company, and fetal bovine serum was purchased from PAN-Biotech; Restriction enzymes (Pme I , Sac II ), rTaq DNA polymerase, T4 DNA ligase purchase since NEB company; DNA Marker, PMD18-T and dNTPs were purchased from TaKaRa Company. Lipofectmine 3000 transfection reagent purchased from Invitrogen company; The plasmid extraction kit was purchased from OMEGA; DNA recovery kit and PCR product purification kit were purchased from Qiagen Company. DNA PCR primers were synthesized by Shanghai Sangon. Anti-human IgG4 Fc (HRP) antibodies, anti-mouse Bcl-2, VEGF-Trap and CD34 antibodies were purchased from Abcam. Antimouse 
$\beta$-actin, caspase-3, BAX, STAT3, AKT, p44/42MAPK(ERK1/2), phospho-STAT3

(named P-STAT3), phospho-AKT (named P-AKT) and phospho-p44/42MAPK(ERK1/2) (named P-ERK1/2) antibodies were purchased from cell signaling technology.

\section{Recombinant Newcastle disease virus}

The rclone30 strain was used to provide a backbone for construction of the recombinant virus. The specific operation steps follow the existing methods in the laboratory. The rNDV viruses were generated as previously described ${ }^{38}$ and sequenced by reverse transcription PCR for fidelity. rNDV and rNDV-VEGF-Trap which express VEGF-Trap were kept in our lab.

\section{Analysis of viral growth in CT26 cell line}

Viral growth was determined in the CT26 cell line. Cells planted in 24-well plates were infected with 0.1 MOI rNDV or rNDV-VEGF-Trap. The supernatants were collected at 12, 24, 36, 48, 60 and $72 \mathrm{~h}$ post infection. The viral concentration was measured at end-point titration on CT26 cells and calculated as 50\% tissue

\section{Expression of VEGF-Trap by tumor cells infected with rNDV-VEGF-Trap}

CT26 cells $\left(5 \times 10^{6}\right.$ cells $)$ were infected with 1 MOI rNDV-VEGF-Trap and rNDV prespectively and the control group received PBS. The methods were accorded to description of Ying An's paper ${ }^{24}$. After $24 \mathrm{~h}$ incubation, cells were collected and washed twice with cold PBS by centrifugation at $500 \times \mathrm{g}$ for $5 \mathrm{~min}$ at $4{ }^{\circ} \mathrm{C}$. The pellet was resuspended in lysis buffer supplemented with proteases inhibitor and the supernatant was stored at $-20{ }^{\circ} \mathrm{C}$. For western blotting analysis, samples were separated by $10 \%$ sodium dodecylsulfate-poly acrylamide gel electrophoresis (SDS-PAGE), and transferred to a nitrocellulose membrane. The blot was visualized by chemiluminescence and autoradiography using X-ray film. Each gel was tested with mouse anti-human IgG4 Fc (HRP) antibody to identify VEGF-Trap.

\section{Cell viability assay}

Cell viability was quantified by a short-term microculture tetrazolium (MTT) assay $^{24}$. Approximately 1-2 × 104 CT26, 4T1, HCT116 and EA.hy926 cells were plated into 96-well plates in complete medium. Then, these cells were infected with rNDV-VEGF-Trap, rNDV at the dose of 0.01 MOI, 0.1 MOI, $1 \mathrm{MOI}$, and $10 \mathrm{MOI}$ in triplicate. According to Ying An's paper ${ }^{24}, 20 \mu \mathrm{L}$ MTT solutions $(5 \mathrm{mg} / \mathrm{mL}$ in sterile phosphate-buffered saline) were added to the cell after $48 \mathrm{~h}$ of incubation. After 4 hours, the MTT solution in the wells was discarded, then $150 \mu \mathrm{L}$ dimethyl sulfoxide (DMSO) was added. The absorption was measured by a microplate reader at $490 \mathrm{~nm}$ (OD490). The cell viability was converted and expressed as the percentage of the control. The cells without any treatment were used as negative control.

Inhibition rate $=($ control group OD - treatment group OD $) /$ control group OD $\times$ $100 \%$

\section{Migration assay}

The effect of rNDV-VEGF-Trap on cell migration was measured by wound healing experiments ${ }^{39}$. The same EA.hy926 cell numbers were respectively seeded into 6-well plates as control and treatment groups. Then, a cell-free area of confluent 
monolayer cells was created by scratching with a $1 \mathrm{~mL}$ pipette tip. After washing the isolated cells, monolayer cells were cultured in a maintenance medium containing $2 \%$ FBS. The cells were incubated with 1 MOI rNDV or rNDV-VEGF-Trap for $24 \mathrm{~h}$ and $48 \mathrm{~h}$, then washed with PBS and photographed. The representative images were captured under 100 magnification with a camera (Nikon, Japan) attached to a microscope. For comparing the migration rate, scratches were photographed in three separate fields, and then the scratch area was measured by ImageJ. The experiment was repeated three times.

\section{Analysis of tumor growth in colon cancer model}

All procedures involving animals were according to the guidelines issued by National Institute of Health and the Institutional Animal Care and Use Committee of Northeast Agriculture University. Healthy 6-week-old female BALB/c mice with a weight of $19 \pm 1 \mathrm{~g}$ were permitted to feed and drink freely. CT26 cells $\left(1 \times 10^{6}\right)$ were subcutaneously implanted into the right groin of mice and tumors were allowed to grow until the average diameter reached 5-8 $\mathrm{mm}$. Reference to previous publications $^{23,24}$, Mice were randomly divided into different groups $(n=10)$, and intratumorally injected with allantoic fluid or $1 \times 10^{7} \mathrm{pfu}$ of the indicated viruses every two day. Tumor volumes were calculated using the following formula: tumor volume $(V)=4 / 3 \times \pi \times S 2 / 2 \times L / 2$, where $S$ is the smallest measured diameter and $L$ is the largest diameter. Animals were humanely culled when tumor size reached 18 $\mathrm{mm}$ in any dimension or at defined experimental time points. After the treatment, animals of each group were sacrificed, their tumors and spleens were excised, weighted, frozen, or fixed in $4 \%$ paraformaldehyde.

\section{Histological analysis and Immunohistochemical (IHC) assay}

According to the method of Elham Assareh paper ${ }^{58}$, the tumor tissues of tumor-bearing mice were fixed with $4 \%$ formalin at room temperature for 2 days, treated with graded concentrations of ethanol and xylene, and embedded in paraffin. The sections of 4-5 mm were mounted on adhesive glass slides. Tumor tissue sections were stained with Hematoxylin and eosin (HE) for investigation of morphological changes in treatment and control groups. IHC staining analysis was performed to localize specific tissue antigens. The sections were incubated at $4{ }^{\circ} \mathrm{C}$ with the primary mouse monoclonal antibodies CD34 (1:2500) for overnight. Antigens is detected with 3,3-diaminobenzidine (DAB). All images were analyzed using Image J software.

\section{Western blot analysis}

CT26 tumor tissues were lysed with RIPA buffer containing protease and phosphatase inhibitors (Beyotime, China) as described by Maryam Farizaneh Behelgardi report ${ }^{59}$. After centrifugation, the protein samples were treated with $5 \times$ loading buffer (Beyotime, China). Protein concentrations were determined by the BCA quantitative kit (Beyotime, China). Equal amounts of total proteins were added into $12 \%$ sodium dodecyl sulfate-polyacrylamide gel for electrophoresis. Proteins were transferred to polyvinyl difluoride (PVDF) membranes followed by blocking with $5 \%$ non-fat milk for $2 \mathrm{~h}$. The primary antibodies including anti-P-AKT, anti-total-AKT, anti-P-STAT3 (1:2000), anti-P-ERK1/2, anti-total-STAT3, anti-total-ERK1/2, anti-P53, anti-BAX, anti-Bcl-2 and anti-caspase-3 (1:1000) were 
incubated overnight at $4{ }^{\circ} \mathrm{C}$. After three times washing in Tris Buffered Saline-Tween (TBS-T), the membranes were incubated with an appropriate HRP-conjugated secondary antibody for $1 \mathrm{~h}$ at room temperature. Protein bands were visualized using ECL reagent.

\section{Animal safety assessment}

Based on the exposure of VEGF-Trap in clinical treatment of various adverse reactions. The diarrhoea, appetite, weight and haemorrhage of the experimental mice were recorded throughout the treatment phase. At the end of the animal experiment, whole blood was collected through the eyeballs of the experimental mice. Then the serum was isolated from whole blood for determination of serum concentrations of AST, ALT and creatinine.

\section{Statistical analysis}

The statistical significance of quantitative data between different groups was determined with GraphPad prism software (Version 5.01, GraphPad Software Inl., La Jolla, California). All data were expressed as mean \pm SEM (standard error of the mean), significance was determined by performing one- or two-sided Student's $t$ tests and defined as a $p$ value $<0.05, p$ value $<0.01$.

\section{Results}

\section{Generation of rNDV-VEGF-Trap}

The VEGF-Trap gene was incorporated at the position between the $\mathrm{P}$ and $\mathrm{M}$ genes of the rNDV genome (Fig. 1A). The incorporated plasmid was transfected into BHK-21 cells. The viral population was amplified by inoculating to the cell cultures and then transfered into 10 days SPF embryo chicken eggs ${ }^{24}$. After 3 days, the allantoic fluid was harvested and analyzed in a HA test ${ }^{24}$. The sucessful generation of rNDV-VEGF-Trap virus were evaluated by the high titers of virus.

\section{Growth and VEGF-Trap protein expression of rNDV-VEGF-Trap}

In order to verify the proliferation ability of rNDV-VEGF-Trap in tumor cells, CT26 cells were infected with 0.1 MOI rNDV or rNDV-VEGF-Trap. The supernatant was harvested at different time points. Then the viral titers in the supernatants were determined in triplicate. As shown in Fig. 1B, compared with the parental virus, rNDV-VEGF-Trap has no significant difference in the kinetics of replication. To investigate the expression of VEGF-Trap protein, CT26 cells were treated with 1 MOI rNDV-VEGF-Trap. Western blotting analysis Fig. 1C showed that the VEGF-Trap expression was detected the tumor cells infected with rNDV-VEGF-Trap.

\section{rNDV-VEGF-Trap suppresses tumor cells and vascular endothelial cells growth}

The direct cytotoxic activity of rNDV-VEGF-Trap was tested in CT26, 4T1, HCT116, EA.hy926 cells in this study. As shown in Fig. 2A, rNDV-VEGF-Trap induced cell apoptosis in a dose-dependent manner during $48 \mathrm{~h}$ treatment. When the cells were infected with rNDV-VEGF-Trap or rNDV of 10 MOI for $48 \mathrm{~h}$, the inhibition rates for EA.hy926 were respectively $85.37 \% \pm 3.20 \%$ and $74.26 \% \pm$ $2.12 \%$. However, there is no significant difference between rNDV and rNDV-VEGF-Trap in inhibiting the growth of the other three cell types. In summary, these data indicate that rNDV-VEGF-Trap significantly inhibits the growth of 
EA.hy926 cells compared with rNDV.

\section{Effects of rNDV-VEGF-Trap on EA.hy926 cell migration}

Scratch test was used to investigate changes in the migration of EA.hy926 cells after treatment with rNDV and rNDV-VEGF-Trap for 48h. As shown in Fig. 2B, the migration rate of EA.hy926 cell in PBS group, rNDV group and rNDV-VEGF-Trap were respectively $81.79 \%, 26.5 \%$ and $12.1 \%$ at $48 \mathrm{~h}$. The results showed that rNDV-VEGF-Trap significantly inhibited EA.hy926 cell migration compared with rNDV.

\section{rNDV-VEGF-Trap suppresses tumor growth in mice}

When the tumor size reached about $100 \mathrm{~mm}^{3}$, the mice were divided into three groups. Their tumor volume and weight were recorded every 2 days. The average tumor size for the model group, rNDV group and rNDV-VEGF-Trap group were $1889.18 \pm 408.16 \mathrm{~mm}^{3}, 728.49 \pm 287.41 \mathrm{~mm}^{3}$ and $351.1 \pm 142.58 \mathrm{~mm}^{3}$ (Fig. 3A). After necropsy, tumors excised from each group showed that rNDV-VEGF-Trap significantly ameliorates tumor texture and vascular density compared to both model and rNDV groups (Fig 3B). Moreover, the average tumor weight of each group were respectively $1.456 \pm 0.459 \mathrm{~g}, 0.603 \pm 0.311 \mathrm{~g}$ and $0.169 \pm 0.154 \mathrm{~g}$ (Fig. 3C). The average rates of tumor/weight in each group were respectively $5.34 \pm 0.81,2.22 \pm$ 0.33 and $0.64 \pm 0.24$ (Fig. 3D). Taken together, these data demonstrate that treatment with rNDV-VEGF-Trap significantly inhibited tumor growth compared to treatment with rDNV in the colon cancer model.

\section{The tumor tissue treated with rNDV-VEGF-Trap show antineoplastic activity and anti-angiogenesis}

As shown in Fig. 4A, H\&E staining tumor sections showed that tumors derived from rNDV-VEGF-Trap demonstrated significant suppression to tumor cell viability than rNDV and model group. Then we evaluated the inhibition of rNDV-VEGF-Trap on vascular endothelial cell proliferation in the tumor tissue by CD34 assay. Compared with model group and rNDV group, we found that the expression of CD34 was significantly decreased by treatment with rNDV-VEGF-Trap (Fig. 4B). These data suggest that rNDV-VEGF-Trap exhibits an antiangiogenic role.

rNDV-VEGF-Trap modulates the related protein expression of angiogensis and apoptosis

To elucidate the antiangiogenic mechanisms of rNDV-VEGF-Trap, the related proteins of VEGF signaling pathway were analyzed by Western blot. As shown in Fig. 5A, rNDV-VEGF-Trap treatment significantly reduced the expression levels of P-AKT, P-ERK and P-STAT3, while there was no difference in expression levels of AKT, ERK, and STAT3. The results showed that rNDV-VEGF-Trap has the ability to inhibit the related protein activation of VEGF/VEGFR downstream signaling pathways. Then the related protein expression of tumor apoptosis were measured by Western blot. As show Fig.5B, rNDV-VEGF-Trap significantly increased the expression levels of P53, BAX and cleaved caspase-3 and significantly downregulated the expression level of Bcl-2 compared with rNDV. The results show that rNDV-VEGF-Trap significantly enhances tumor apoptosis. These data suggest that rNDV-VEGF-TrapVEGF further promotes apoptosis through anti-angiogenesis, 
consequently inhibiting the growth of tumor tissue.

\section{rNDV-VEGF-Trap demonstrates therapeutic safety}

Previous papers demonstrate that adminstraion of VEGF-Trap exhibits common adverse events in clinical, including diarrhoea, decreased appetite, weight loss, haemorrhage, deteriorated creatinine levels, AST and ALT. In this study, our results showed these common adverse events were not significantly observed in each group (normal, model, rNDV, rNDV-VEGF-Trap) of the CT26-bearing mice (Table1, Fig. 6 ). These results suggest that rNDV-VEGF-Trap exhibits therapeutic safety.

\section{Discussion}

As one of the oncolytic viruses, NDV represents a new class of therapeutic agents of cancer therapy ${ }^{40}$. Some clinical trials of phase I-IV colon cancer based on NDV vectors have shown encouraging results ${ }^{41}$. At present, VEGF-Trap, bevacizumab, cetuximab and panitumumab show promising in antiangiogenic therapy, in which VEGF-Trap has the advantages of significant therapeutic effect and long Half-Life compared with others ${ }^{42,43}$. However, VEGF-Trap inevitably arises multiple adverse effects in the clinical treatment of colon cancer ${ }^{37}$. Previous study showed that researchers used recombinant AAV2 as a delivery vehicle to achieve long-lasting expression of VEGF-Trap protein in a mouse model, simultaneously suppressing primary tumor growth and preventing the metastases of tumors ${ }^{44}$. However, the therapeutic effect of AAV2 vector was not significant. These problems indicate that it is important for reducing the adverse reactions of VEGF-Trap and enhancing colon cancer apoptosis and anti-angiogenesis through VEGF-Trap gene was integrated into NDV genes.

In this study, we generated rNDV-VEGF-Trap using reverse genetics. As anticipated, the kinetics of replication of rNDV-VEGF-Trap did not show a significant change and rNDV-VEGF-Trap successfully expressed VEGF-Trap protein in the CT26 tumor cells. MTT results showed that the EA.hy926 cells were infected with the virus at 10 MOI for $48 \mathrm{~h}$, the apoptosis rate induced by rNDV-VEGF-Trap was $85.37 \%$ and rNDV was $74.26 \%$. The results showed that rNDV-VEGF-Trap enhanced pro-apoptotic activity of the virus. Scratch test results show that the EA.hy926 cells were infected with the virus at $1 \mathrm{MOI}$ for $48 \mathrm{~h}$, the relative mobility rate induced by rNDV-VEGF-Trap was $12.1 \%$ and rNDV was $26.5 \%$. The results showed that rNDV-VEGF-Trap enhanced the ability of the virus to inhibit the migration of EA.hy926 cells. The results are similar to the previously report that VEGF-Trap protein inhibits vascular endothelial cell proliferation and migration ${ }^{36}$. In summary, rNDV expressing VEGF-trap was successfully generated in this study. In vitro, VEGF-Trap protein expressed from rNDV-VEGF-Trap inhibits the proliferation and migration of vascular endothelial cells.

Based on the encouraging in vitro data, we investigated the therapeutic efficacy of rNDV-VEGF-Trap in CT26-bearing mice. The results show that treatment with rNDV-VEGF-Trap reduced tumor volume of CT26-bearing mice by more than 3 folds and tumor weight by more than 4 folds compared to rNDV. The results indicate that rNDV-VEGF-Trap effectively inhibits tumor growth. Previous studies have shown 
that tumor tissue angiogenesis is caused by excessive and abnormal proliferation of vascular endothelial cells ${ }^{45,46}$. As surface markers of vascular endothelial cells, CD34 is commonly applied to detect the situation of vascular proliferation 47,48 . Immunohistochemistry results of CD34 showed rNDV-VEGF-Trap significantly reduced the number of vascular endothelial cells in the tumor tissues of the tumor-bearing mice. The results indicate that rNDV-VEGF-Trap has a siginificant antiangiogenic ability in tumor tissue than rNDV. In summary, rNDV-VEGF-Trap significantly enhances the inhibition effect on the tumor growth of the CT26 tumor-bearing mice and VEGF-Trap protein expressed by rNDV-VEGF-Trap effectively inhibits the proliferation of vascular endothelial cells in tumor tissues.

Based on the results of in vivo experiments, we have shown that rNDV-VEGF-Trap has the ability to inhibit tumor growth and angiogenesis in the tumor. However, the mechanism of rNDV-VEGF-Trap inhibiting angiogenesis and tumor growth is not clear. Previous studies have shown that VEGF-Trap binds VEGF and prevents its binding to and activation of $\mathrm{VEGFR}^{36}$. As a pivotal and pervasive pro-angiogenic factor, VEGF regulates multiple facets of tumor angiogenesis through VEGFR $^{49}$. In response to its binding, VEGFR initiates signaling through the Raf-MEK-ERK, PI3K-AKT kinase cascades ${ }^{50-52}$ and STAT3 ${ }^{53}$. Signaling through these pathways promotes proliferation, survival and chemotaxis in endothelial cells and ultimately produces the characteristic effects of VEGF on vessels, such as increased vascular permeability and angiogenesis ${ }^{54,55}$. In general, these signaling pathways in endothelial cells are not activated in normal mouse organs, but only in blood vessels of tumor tissues. In addition, these signaling pathways were activated not only by VEGF-VEGFR, but also by other angiogenic promoters (such as basic FGF) and thus, may serve as general indicators of angiogenic activation ${ }^{56,57}$. To determine these signaling pathways are blocked in tumor vessels in vivo by rNDV-VEGF-Trap, we employed Western blot for detection of ERK (P-ERK), AKT (P-AKT) and STAT3 (P-STAT3) in tumor tissue in mice. The results showed that treatment with rNDV-VEGF-Trap significantly reduced the phosphorylation levels of AKT, ERK and STAT3 compared with rNDV or PBS. This suggests that rNDV-VEGF-Trap inhibits tumor tissue angiogenesis by blocking the activation of these signaling pathways. Previous studies demonstrated that VEGF-Trap indirectly induces apoptosis of tumor cells by inhibiting angiogenesis ${ }^{36}$. In order to demonstrate that rNDV-VEGF-Trap enhances virus-mediated tumor tissue apoptosis, we detected the expression levels of P53, BAX, Bcl-2 and cleaved caspase-3 in tumor tissues by Western blotting. The results showed that the expression levels of P53, BAX, and cleaved caspase-3 in rNDV-VEGF-Trap treated tumor tissues were significantly increased and the expression levels of Bcl-2 were significantly decreased compared with rNDV. This indicates that rNDV-VEGF-Trap significantly promotes the apoptosis of tumor tissue. In this part, we preliminarily confirmed the mechanism by which rNDV-VEGF-Trap inhibits angiogenesis and tumor growth. First, rNDV-VEGF-Trap inhibits the activation of AKT, ERK and STAT3 signaling pathways by blocking VEGF and VEGFR binding, leading to a reduced tumor angiogenesis. Secondly, the VEGF-Trap effectively enhanced the viral-vector- 
mediated tumor tissue apoptosis by inducing the expression of apoptotic proteins P53, BAX, and cleaved caspase-3.

In previous studies, purified VEGF-Trap proteins were applied to cancer therapy, and the main problem was the side effects ${ }^{37}$. In order to evaluate the adverse reactions of rNDV-VEGF-Trap in vivo, a toxicity study was performed. We have detected the AST, creatinine, ALT, diarrhoea, appetite, weight and haemorrhage of the experimental mice. The results showed that these parameters in rNDV-VEGF-Trap treated mice were all within the normal range, indicating that rNDV-VEGF-Trap was relatively safe in the treatment of colon cancer.

In conclusion, our study shows that rNDV-VEGF-Trap can effectively inhibit the tumor growth and angiogenesis of the CT26-bearing mice. Moreover, treatment with rNDV-VEGF-Trap reduced the phosphorylation levels of AKT, ERK1/2 and STAT3, increased the expression levels of P53, BAX and cleaved caspase-3 in the tumor tissue. In addition, rNDV-VEGF-Trap effectively reduces the adverse reactions induced by the treatment of purified VEGF-Trap. Thereby, this study provides a potential candidate for the treatment of colon cancer.

\section{Availability of data and materials}

The data analyzed during the current study may be available upon reasonable request.

\section{Ethics statement}

This study was approved by the ethics committee of Northeast Agriculture University. All experimental protocols involving animals followed the guidelines issued by National Institute of Health and the Institutional Animal Care and Use Committee of Northeast Agriculture University.

\section{Conflicts interest}

The authors declare that there are no conflicts of interest.

\section{Achnowledgements}

This work was supported by a grant from the major prophase project of Heilongjiang development and reform commission ([2011]1570), education department of Heilongjiang province (TSTAU-R2018017) and National Key R\&D program of China (2017YFD0501102, 2017YFD0501103-03).

\section{Author' contributions statement}

FR, M. YK, C performed the research, and analyzed the data. H, S. LM, T and JR, Y participated in data collection and analysis. W, $\mathbf{X}$ and DS, Li contributed to the initial and consequent project discussion, manuscript discussion and revision. All the authors approved the final version of the manuscript. 


\section{Author' information}

${ }^{1}$ Northeast Agricultural University, Harbin, China

${ }^{2}$ Jiangsu Kanion Pharmaceutical CO. LTD, Jiangsu, Lianyungang. State Key Laboratory of New-tech for Chinese Medicine Pharmaceutical Process, Jiangsu, China

Fanrui Meng Northeast Agricultural University, Harbin, China $1192841993 @$ qq.com Yukai Cao Northeast Agricultural University, Harbin, China 1179778594@qq.com Han Su Northeast Agricultural University, Harbin, China 727690458@qq.com Limin Tian Northeast Agricultural University, Harbin, China 2676690763@qq.com Jiarui Yang Jiangsu Kanion Pharmaceutical CO. LTD, Jiangsu, Lianyungang. State Key Laboratory of New-tech for Chinese Medicine Pharmaceutical Process, Jiangsu, China1260481892@qq.com

Wei Xiao Jiangsu Kanion Pharmaceutical CO. LTD, Jiangsu, Lianyungang. State Key Laboratory of New-tech for Chinese Medicine Pharmaceutical Process, Jiangsu, China xw_kanion@163.com (second corresponding author)

Deshan Li Northeast Agricultural University, Harbin, China deshanli@163.com (first corresponding author)

*Corresponding authors at: School of Life Science, Northeast Agricultural University, Harbin, Heilongjiang Province, 150030, China, E-mail addresses: deshanli@163.com (DS, Li first corresponding author).

**Corresponding authors at: Jiangsu Kanion Pharmaceutical CO. LTD, Jiangsu, Lianyungang. State Key Laboratory of New-tech for Chinese Medicine Pharmaceutical Process, Jiangsu, 220000, China. E-mail addresses: xw_kanion@163.com ( W, Xiao second corresponding author)

\section{References}

1 Siegel, R. L., Miller, K. D. \& Jemal, A. Cancer Statistics, 2017. CA Cancer J Clin 67, 7-30, doi:10.3322/caac.21387 (2017).

2 Wang, W. et al. Molecular subtyping of colorectal cancer: Recent progress, new challenges and emerging opportunities. Semin Cancer Biol 55, 37-52, doi:10.1016/j.semcancer.2018.05.002 (2019).

3 Liu, H. et al. Anti-tubulin agent vinorelbine inhibits metastasis of cancer cells by regulating epithelial-mesenchymal transition. Eur J Med Chem 200, 112332, doi:10.1016/j.ejmech.2020.112332 (2020).

4 Yuan, L. et al. Resveratrol inhibits the invasion and metastasis of colon cancer through reversal of epithelial mesenchymal transition via the AKT/GSK3beta/Snail signaling pathway. Mol Med Rep 20, 2783-2795, doi:10.3892/mmr.2019.10528 (2019).

5 Stewart, C. L. et al. Cytoreduction for colorectal metastases: liver, lung, peritoneum, lymph nodes, bone, brain. When does it palliate, prolong survival, and potentially cure? Curr Probl Surg 55, 330-379, doi:10.1067/j.cpsurg.2018.08.004 (2018).

6 Zugazagoitia, J. et al. Current Challenges in Cancer Treatment. Clin Ther 38, 1551-1566, doi:10.1016/j.clinthera.2016.03.026 (2016). 
4717 Masi, G. et al. Liver metastases from colorectal cancer: how to best

472 complement medical treatment with surgical approaches. Future Oncology 7, 1299-1323, doi:10.2217/fon.11.108 (2011).

8 Esther Ua Cidón. The Challenge of Metastatic Colorectal Cancer. Clinical Medicine Insights: Oncology 4, 55-60, doi:10.4137/CMO.S5214 (2010).

Shi, G. et al. Modulating the Tumor Microenvironment via Oncolytic Viruses and CSF-1R Inhibition Synergistically Enhances Anti-PD-1 Immunotherapy. Mol Ther 27, 244-260, doi:10.1016/j.ymthe.2018.11.010 (2019).

11 Sarvizadeh, M. et al. Vaccines for colorectal cancer: an update. J Cell Biochem 120, 8815-8828, doi:10.1002/jcb.28179 (2019).

12 Weijing, Y. et al. In Situ Dendritic Cell Vaccine for Effective Cancer Immunotherapy. ACS nano vol 13, 3083-3094, doi:10.1021/acsnano.8b08346 (2019)

13 Lazarus, J. et al. Spatial and phenotypic immune profiling of metastatic colon cancer. JCI Insight 3, doi:10.1172/jci.insight.121932 (2018).

14 Gordon, S. R. et al. PD-1 expression by tumour-associated macrophages inhibits phagocytosis and tumour immunity. Nature 545, 495-499, doi:10.1038/nature22396 (2017). Treatment of Colon and Rectal Malignancies. Cancer Invest 37, 393-414, doi:10.1080/07357907.2019.1660887 (2019).

16 Wang, Y. et al. Newcastle disease virus induces G0/G1 cell cycle arrest in asynchronously growing cells. Virology 520, 67-74, doi:10.1016/j.virol.2018.05.005 (2018).

17 Afonso, C. L. et al. Taxonomy of the order Mononegavirales: update 2016. Arch Virol 161, 2351-2360, doi:10.1007/s00705-016-2880-1 (2016).

18 Ramp, K. et al. Coexpression of avian influenza virus H5 and N1 by recombinant Newcastle disease virus and the impact on immune response in chickens. Avian Dis 55, 413-421, doi:10.1637/9652-011111-Reg.1 (2011).

19 Liu, H., de Almeida, R. S., Gil, P. \& Albina, E. Comparison of the efficiency of different newcastle disease virus reverse genetics systems. J Virol Methods 249, 111-116, doi:10.1016/j.jviromet.2017.08.024 (2017).

20 Kim, S. H. \& Samal, S. K. Newcastle Disease Virus as a Vaccine Vector for Development of Human and Veterinary Vaccines. Viruses 8, doi:10.3390/v8070183 (2016).

21 Fountzilas, C., Patel, S., \& Mahalingam, D. Review: Oncolytic virotherapy, updates and future directions. Oncotarget 8, 102617-102639, doi: /10.18632/oncotarget.18309 (2017).

22 Zhang, S., Sun, Y., Chen, H., Dai, Y., Zhan, Y., Yu, S., Qiu, X., Tan, L., Song, C., \& Ding, C. Activation of the PKR/eIF2 $\alpha$ signaling cascade inhibits replication of Newcastle disease virus. Virology journal 11, 62, doi:/10.1186/1743-422X-11-62 (2014). 
23 Bai, F. L. et al. Genetically engineered Newcastle disease virus expressing interleukin-2 and TNF-related apoptosis-inducing ligand for cancer therapy. Cancer Biol Ther 15, 1226-1238, doi:10.4161/cbt.29686 (2014).

24 Ying An et al. Recombinant Newcastle disese virus expressing P53 demonstrates promising antitumor efficiency in hepatoma model. Journal of Biomedical Science 14, 1247-1258, doi: /10.1186/s12929-016-0273-0 (2016).

25 Al-Shammari, A. M., Rameez, H. \& Al-Taee, M. F. Newcastle disease virus, rituximab, and doxorubicin combination as anti-hematological malignancy therapy. Oncolytic Virother 5, 27-34, doi:10.2147/OV.S95250 (2016).

26 Tysome, J. R., Lemoine, N. R. \& Wang, Y. Update on oncolytic viral therapy targeting angiogenesis. Onco Targets Ther 6, 1031-1040, doi:10.2147/OTT.S46974 (2013).

27 Jaszai, J. \& Schmidt, M. H. H. Trends and Challenges in Tumor Anti-Angiogenic Therapies. Cells 8, doi:10.3390/cells8091102 (2019).

28 Li, X. et al. The Tumor Vessel Targeting Strategy: A Double-Edged Sword in Tumor Metastasis. Cells 8, doi:10.3390/cells8121602 (2019).

29 Amerizadeh, F., Khazaei, M., Maftouh, M., Mardani, R. \& Bahrami, A. miRNA Targeting Angiogenesis as a Potential Therapeutic Approach in the Treatment of Colorectal Cancers. Curr Pharm Des 24, 4668-4674, doi:10.2174/1381612825666190110161843 (2018).

30 Battaglin, F. et al. The role of tumor angiogenesis as a therapeutic target in colorectal cancer. Expert Rev Anticancer Ther 18, 251-266, doi:10.1080/14737140.2018.1428092 (2018).

31 Lopez-Ocejo, O., Viloria-Petit, A., Bequet-Romero, M., Mukhopadhyay, D., Rak, J., \& Kerbel, R. S. Oncogenes and tumor angiogenesis: the HPV-16 E6 oncoprotein activates the vascular endothelial growth factor (VEGF) gene promoter in a p53 independent manner. Oncogene 19, 4611-4620, doi:10.1038/sj.onc.1203817 (2000).

32 Rak, J., Yu, J. L., Klement, G., \& Kerbel, R. S. Oncogenes and angiogenesis: signaling three-dimensional tumor growth. The journal of investigative dermatology. Symposium proceedings $\quad 5$ 24-33, doi:10.1046/j.1087-0024.2000.00012.x (2000).

33 Chung, A. S. \& Ferrara, N. Developmental and pathological angiogenesis. Annu Rev Cell Dev Biol 27, 563-584, doi:10.1146/annurev-cellbio-092910-154002 (2011).

34 Seeber, A., Gunsilius, E., Gastl, G. \& Pircher, A. Anti-Angiogenics: Their Value in Colorectal Cancer Therapy. Oncol Res Treat 41, 188-193, doi:10.1159/000488301 (2018).

35 Holash, J., Davis, S., Papadopoulos, N. et al. VEGF-Trap: a VEGF blocker with potent antitumor effects. Proceedings of the National Academy of Sciences of the United States of America 99, 11393-11398, doi:10.1073/pnas.172398299 (2002).

36 Lassoued, W. et al. Effect of VEGF and VEGF Trap on vascular endothelial cell signaling in tumors. Cancer Biol Ther 10, 1326-1333, 
doi:10.4161/cbt.10.12.14009 (2010).

37 Ivanova, J. I. et al. Real-world treatment patterns and effectiveness among patients with metastatic colorectal cancer treated with ziv-aflibercept in community oncology practices in the USA. Med Oncol 34, 193, doi:10.1007/s12032-017-1049-4 (2017).

38 Wu, Y. et al. Recombinant Newcastle disease virus Anhinga strain (NDV/Anh-EGFP) for hepatoma therapy. Technol Cancer Res Treat 13, 169-175, doi:10.7785/tcrt.2012.500356 (2014).

39 Yin, L. et al. Veratramine suppresses human HepG2 liver cancer cell growth in vitro and in vivo by inducing autophagic cell death. Oncol Rep 44, 477-486, doi:10.3892/or.2020.7622 (2020).

40 Schirrmacher, V., van Gool, S. \& Stuecker, W. Breaking Therapy Resistance: An Update on Oncolytic Newcastle Disease Virus for Improvements of Cancer Therapy. Biomedicines 7, doi:10.3390/biomedicines7030066 (2019).

41 Taguchi, S., Fukuhara, H., Homma, Y. \& Todo, T. Current status of clinical trials assessing oncolytic virus therapy for urological cancers. Int J Urol 24, 342-351, doi:10.1111/iju.13325 (2017).

42 Zirlik, K. \& Duyster, J. Anti-Angiogenics: Current Situation and Future Perspectives. Oncol Res Treat 41, 166-171, doi:10.1159/000488087 (2018).

43 Modest, D. P., Pant, S. \& Sartore-Bianchi, A. Treatment sequencing in metastatic colorectal cancer. Eur J Cancer 109, 70-83, doi:10.1016/j.ejca.2018.12.019 (2019).

44 Lu, L. et al. AAV2-mediated gene transfer of VEGF-Trap with potent suppression of primary breast tumor growth and spontaneous pulmonary metastases by long-term expression. Oncol Rep 28, 1332-1338, doi:10.3892/or.2012.1915 (2012).

45 Ahn, J. B. et al. Circulating endothelial progenitor cells (EPC) for tumor vasculogenesis in gastric cancer patients. Cancer Lett 288, 124-132, doi:10.1016/j.canlet.2009.06.031 (2010).

46 Greenfield, J. P., Cobb, W. S. \& Lyden, D. Resisting arrest: a switch from angiogenesis to vasculogenesis in recurrent malignant gliomas. J Clin Invest 120, 663-667, doi:10.1172/JCI42345 (2010).

47 Sasano, H., \& Suzuki, T. Pathological evaluation of angiogenesis in human tumor. Biomedicine \& pharmacotherapy 59, S334-S336. doi: 10.1016/s0753-3322(05)80068-x (2005).

48 Nai, Q. et al. Primary Small Intestinal Angiosarcoma: Epidemiology, Diagnosis and Treatment. J Clin Med Res 10, 294-301, doi:10.14740/jocmr3153w (2018).

49 Olsson, A. K., Dimberg, A., Kreuger, J. \& Claesson-Welsh, L. VEGF receptor signalling - in control of vascular function. Nat Rev Mol Cell Biol 7, 359-371, doi:10.1038/nrm1911 (2006).

50 Suzuma, K. et al. Vascular endothelial growth factor induces expression of connective tissue growth factor via KDR, Flt1, and phosphatidylinositol 3-kinase-akt-dependent pathways in retinal vascular cells. J Biol Chem 275, 
40725-40731, doi:10.1074/jbc.M006509200 (2000).

51 Gerber, H. P. et al. Vascular endothelial growth factor regulates endothelial cell survival through the phosphatidylinositol 3'-kinase/Akt signal transduction pathway. Requirement for Flk-1/KDR activation. $J$ Biol Chem 273, 30336-30343, doi:10.1074/jbc.273.46.30336 (1998).

52 Trinh, X. B. et al. The VEGF pathway and the AKT/mTOR/p70S6K1 signalling pathway in human epithelial ovarian cancer. $\mathrm{Br} J$ Cancer 100 , 971-978, doi:10.1038/sj.bjc.6604921 (2009).

53 Chen, S. H. et al. Activated STAT3 is a mediator and biomarker of VEGF endothelial activation. Cancer Biol Ther 7, 1994-2003, doi:10.4161/cbt.7.12.6967 (2008).

54 Shibuya, M. \& Claesson-Welsh, L. Signal transduction by VEGF receptors in regulation of angiogenesis and lymphangiogenesis. Exp Cell Res 312, 549-560, doi:10.1016/j.yexcr.2005.11.012 (2006).

55 Nagy, J. A., Benjamin, L., Zeng, H., Dvorak, A. M. \& Dvorak, H. F. Vascular permeability, vascular hyperpermeability and angiogenesis. Angiogenesis 11, 109-119, doi:10.1007/s10456-008-9099-z (2008).

56 Shono, T., Kanetake, H. \& Kanda, S. The role of mitogen-activated protein kinase activation within focal adhesions in chemotaxis toward FGF-2 by murine brain capillary endothelial cells. Exp Cell Res 264, 275-283, doi:10.1006/excr.2001.5154 (2001).

57 Holnthoner, W., Kerenyi, M., Groger, M., Kratochvill, F. \& Petzelbauer, P. Regulation of matrilysin expression in endothelium by fibroblast growth factor-2. Biochem Biophys Res Commun 342, 725-733, doi:10.1016/j.bbrc.2006.02.011 (2006).

58 Elham Assareh et al. A cyclic petide reproducing the helix of VEGF-B binds to VEGFR-1 and VGEFR-2 and inhibits angiogenesis and tumor growth. Biochemical Journal. 25, 361-367. doi.org/10.1042/BCJ20180823 (2018)

59 Maryam Farzaneh Behelgardi, Saber Zahri et al. A peptide mimicking the binding sites of VEGF-A and VEGF-B inhibits VEGF-R1/2 drive angiogenesis, tumor growth and metasis. Scientific Reports. 8:17924. doi:10.1038/s41598-018-36394-0 (2018).

\section{Figure}

\section{Table1}

Diarrhoea, appetite, weight and haemorrhage situation of CT26-bearing mice throughout the treatment phase.

\begin{tabular}{lcccc}
\hline & Normal & Model & rNDV & rNDV-VEGF-Trap \\
\hline Diarrhoea & no & no & no & no \\
Decreased appetite & no & no & no & no \\
Weight $(\mathrm{g})$ & $26 \pm 1.3$ & $25 \pm 0.5$ & $24 \pm 1.6$ & $25 \pm 0.8$ \\
Haemorrhage & no & no & no & no \\
\hline
\end{tabular}


Data was presented from ten mice per group. no: No clinical symptoms were observed 642 during the experimental period.

A

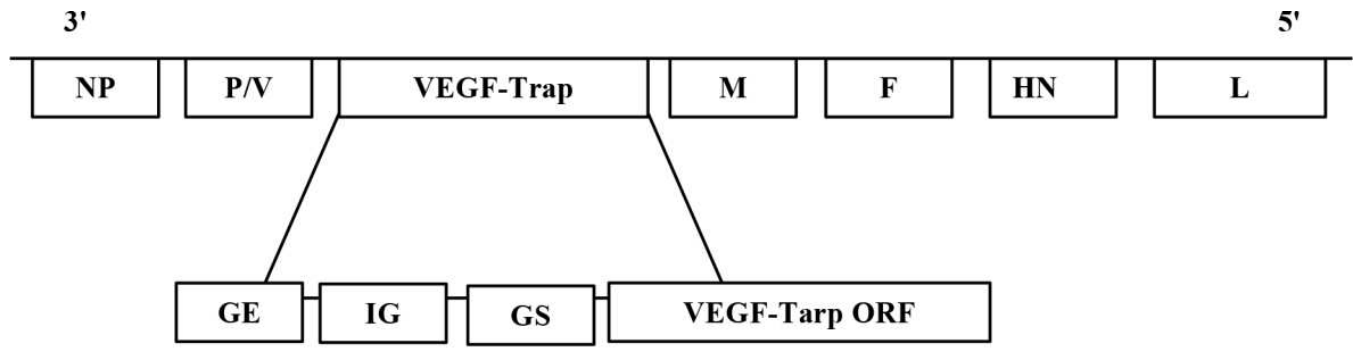

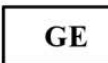

IG

TTAAGAAAAAA

T

GS ACGGGTAGAAG

B

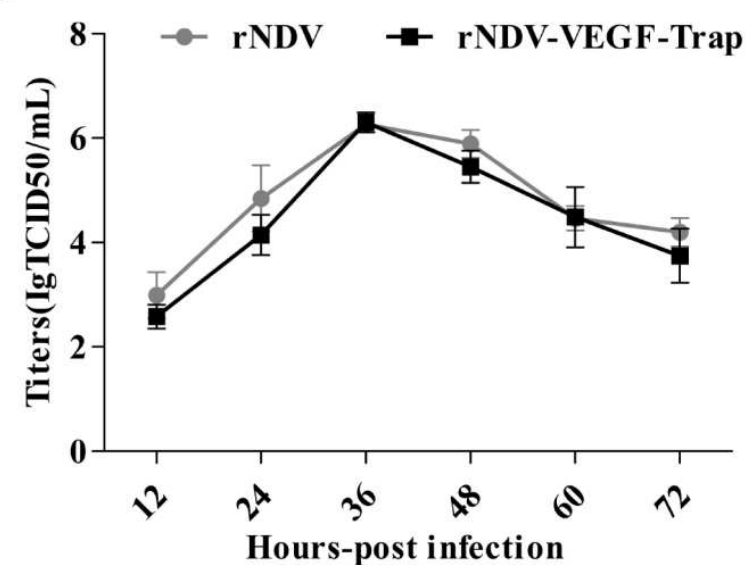

C

VEGF-Trap

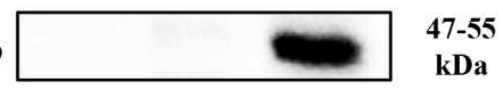

643

Hours-post infection

644

645

646

647

648

649

650

651

652

653

654

Fig.1 Schematic representation of the genomes, characterization and VEGF-Trap protein of rNDV-VEGF-Trap (A) Diagram showed insertion of the VEGF-Trap gene into the NDV genomer at the position between the P and M genes. (B) Growth curves of rNDV-VEGF-Trap. CT26 cells were infected with rNDV and rNDV-VEGF-Trap at 0.1 MOI. Cell monolayers were lysed at 12, 24, 48, 60 and 72h post-infection for intracellular titer measurement by TCID50. (C) The expression of VEGF-Trap proteins in CT26 cells. CT26 cells were respectively infected with 1 MOI rNDV-VEGF-Trap or rNDV and the control group received PBS. After 24h incubation, cells were washed twice with cold PBS and collected by centrifugation at $500 \times \mathrm{g}$ for $5 \mathrm{~min}$ at $4{ }^{\circ} \mathrm{C}$. The cells were resuspended in lysis buffer supplemented with proteases inhibitor and after centrifugal the supernatant was used to western blot. 

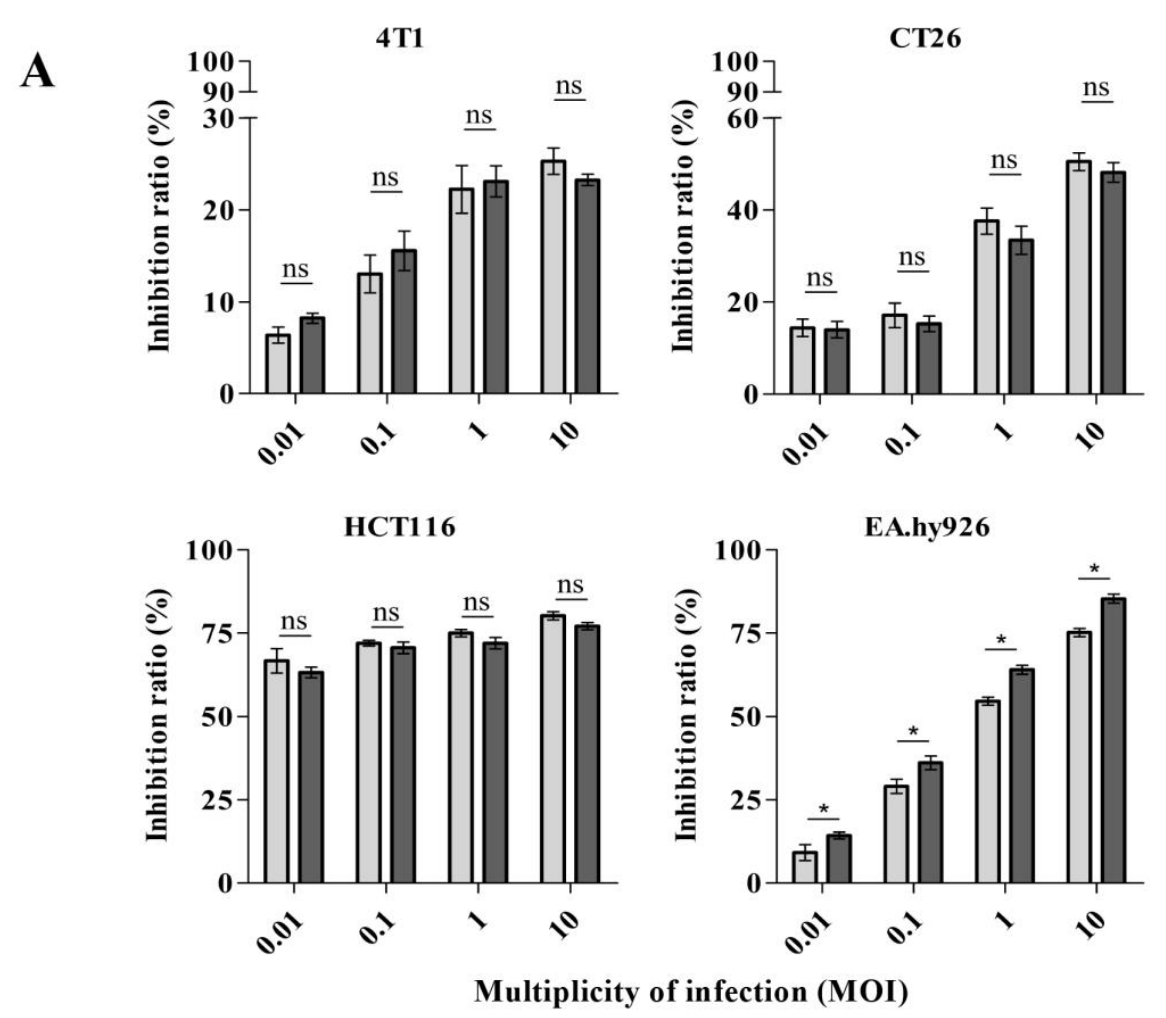

Multiplicity of infection (MOI)

$\square$ rNDV $\square$ rNDV-VEGF-Trap

\section{B}

PBS RNDV rNDV-VEGF-

$\mathbf{0 h}$

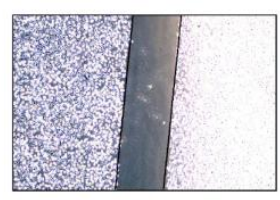

$24 \mathrm{~h}$
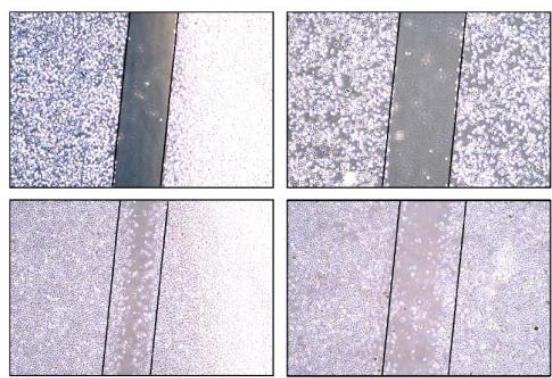

Trap
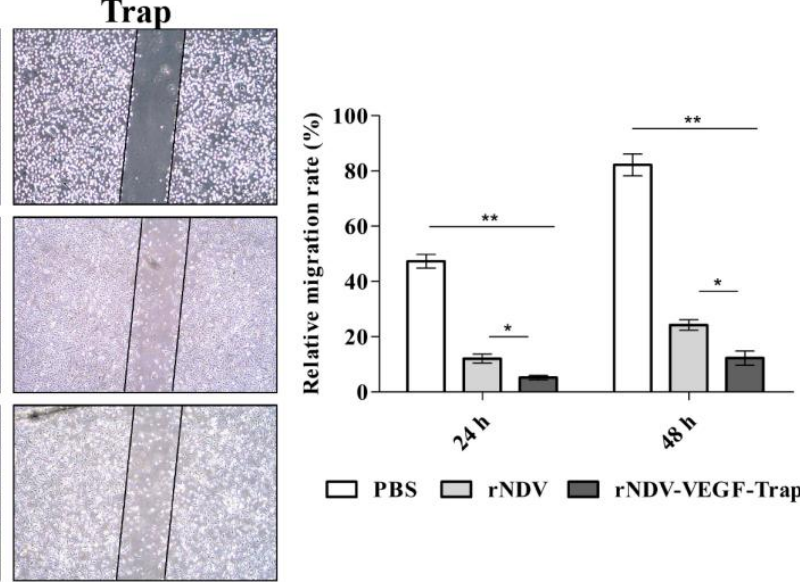

$48 \mathrm{~h}$
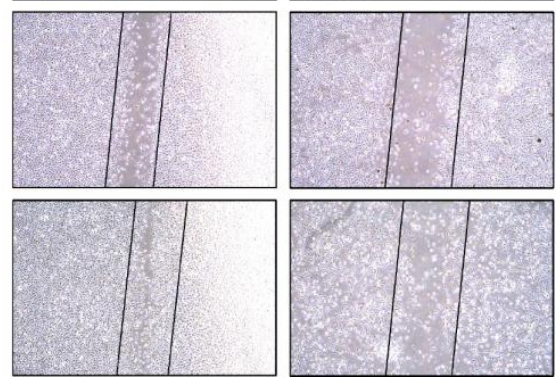

PBS rNDV rNDV-VEGF-Trap

Fig.2 In vitro studies (A) Cytotoxic effects of rNDV-VEGF-Trap on cancer cells and vascular endothelial cells. 4T-1, CT26, HCT116, and EA.hy926 cells incubated with PBS were used as controls. MTT method was used to measure the cell density. The data are the means \pm SEM. of triple samples $(* P<0.05$; $* * P<0.01)$. (B) Inhibition of cell migration by recombinant virus. Wound healing assay was performed on EA.hy926 cells, the cells were infected with 1 MOI rNDV-VEGF-Trap or rNDV and cell migration was evaluated at regular time points post-infection. The number of migrated cells were assessed by microscope. The assay was performed in triplicate. The data are the means \pm SEM of triple samples (ns, no significant; $* P<0.05$; $* * P<$ $0.01)$. 
A
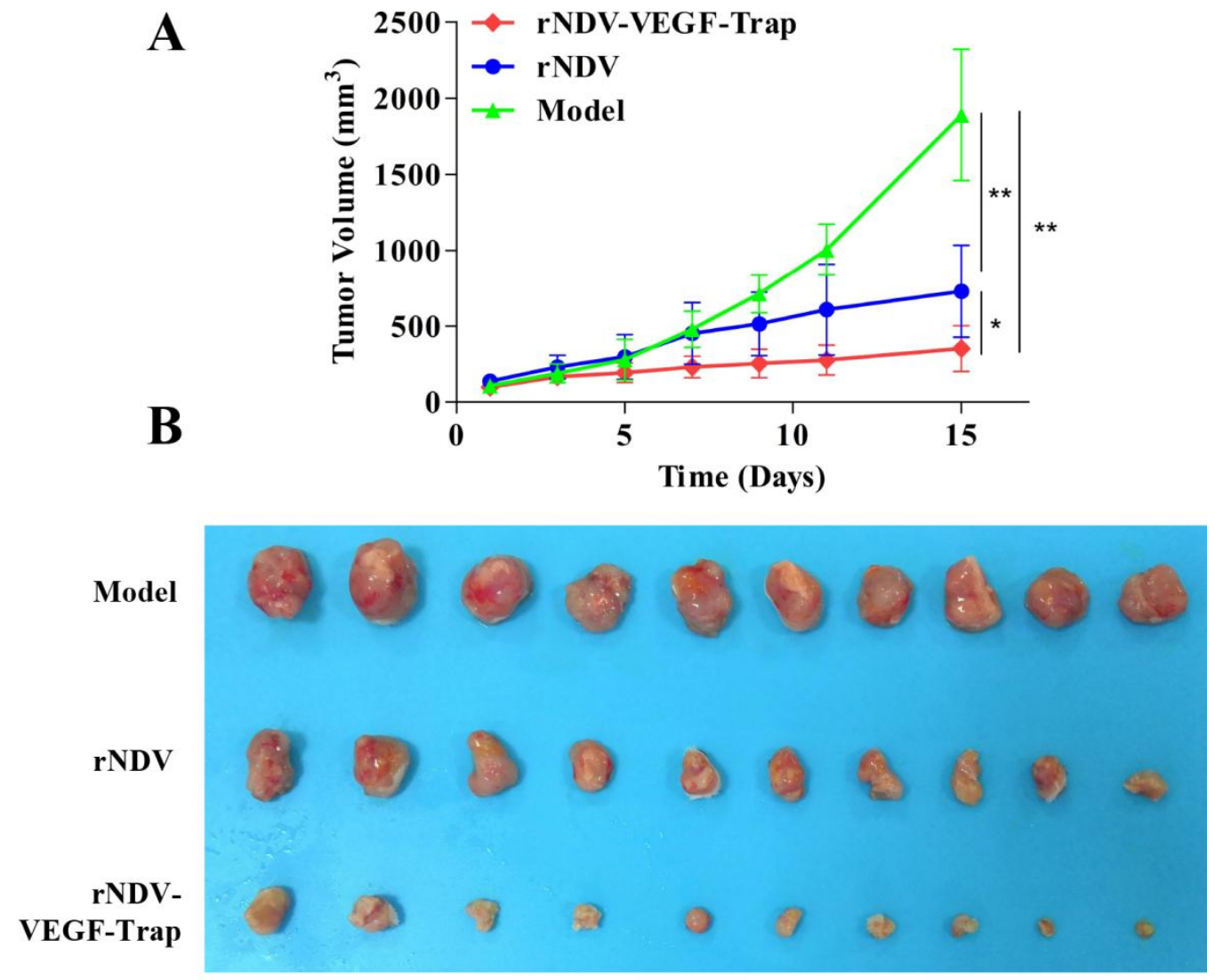

C

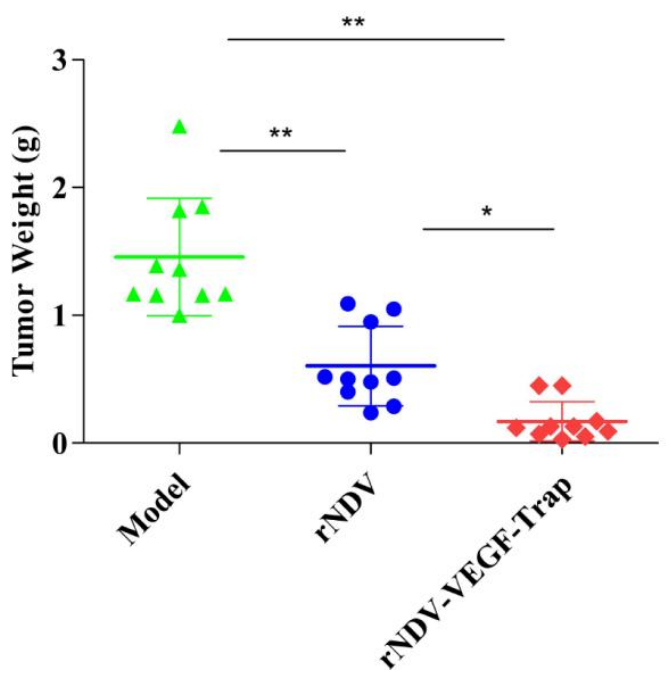

D

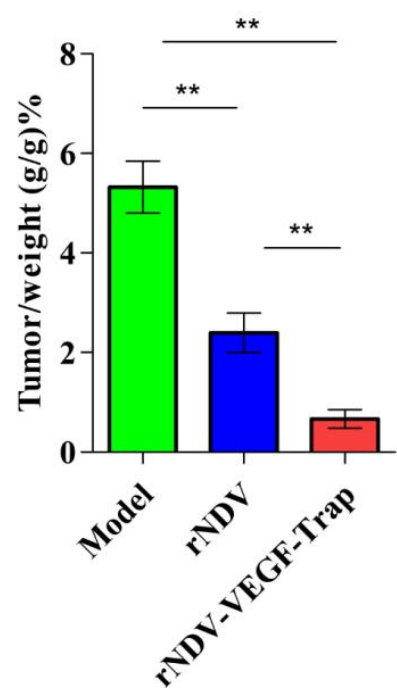

Fig.3 rNDV-VEGF-Trap enhances inhibition of tumor growth of CT26-bearing mice. Six-week-old female BALB/c mice were injected with $10^{6} \mathrm{CT} 26$ cells in the right groin. When the tumor size reached about $100 \mathrm{~mm}^{3}$, the mice were intratumorally treated with rNDV or rNDV-VEGF-Trap at $10^{7} \mathrm{pfu}$. The mice were euthanized and necropsy was performed after 15 days of treatment. (A) The tumor volume of CT26-bearing mice was measured in two dimensions every other day using digital calipers. (B) Tumor collected in the end of the experiment. (C) Tumor Weight. (D) Tumor to body weight ratio. Model: mice treated with allantoic fluid. $n=10$; ns, no significant; $* P<0.05 ; * * P<0.01$. 

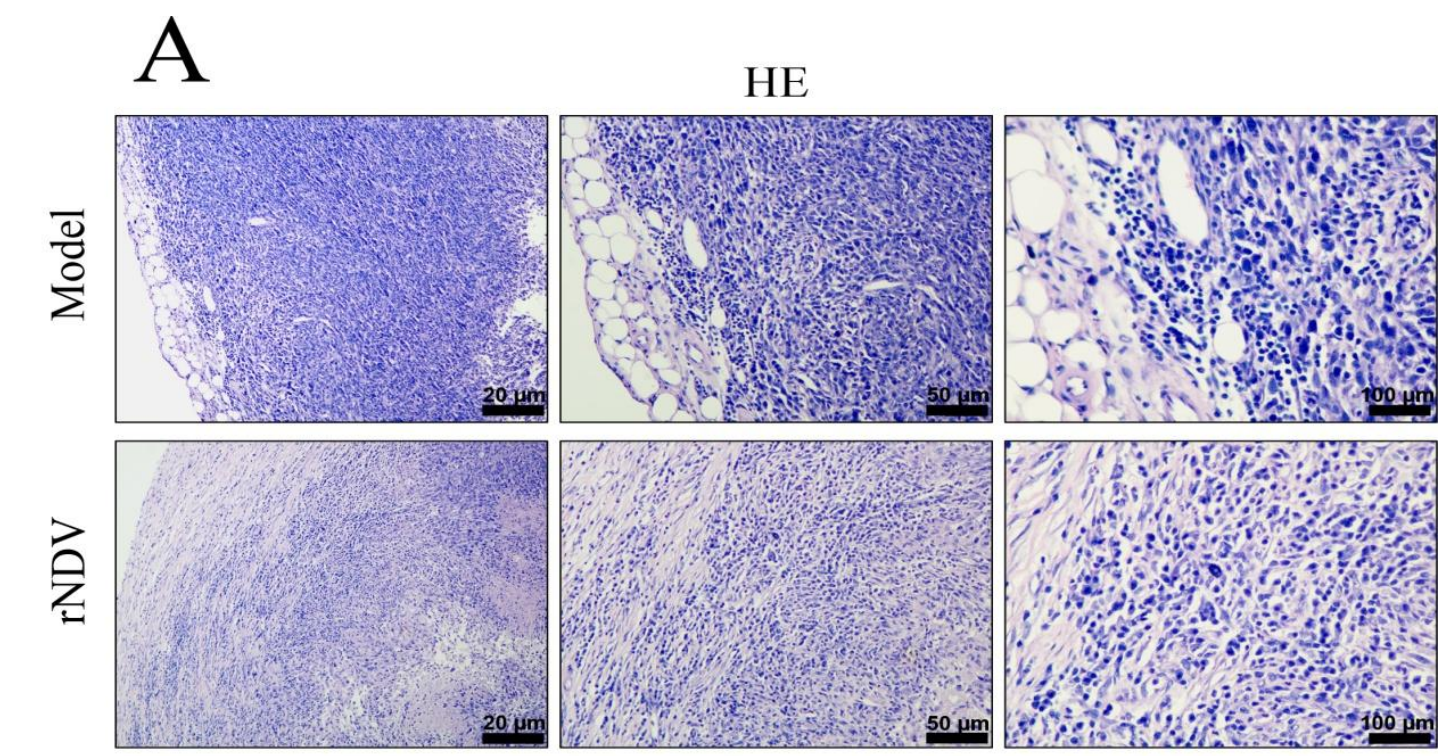

676
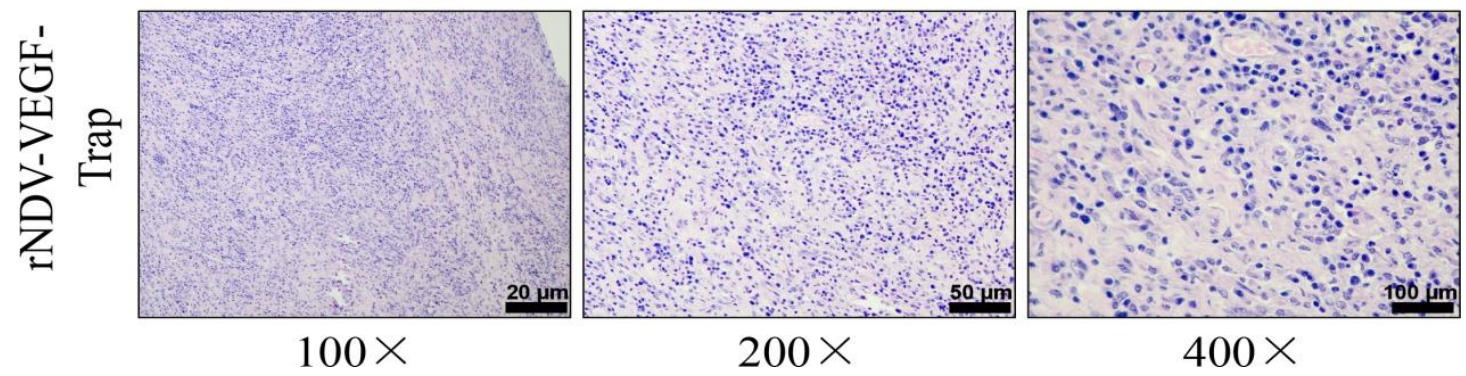

B

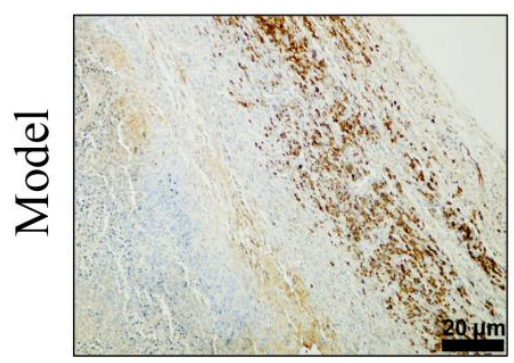

CD34
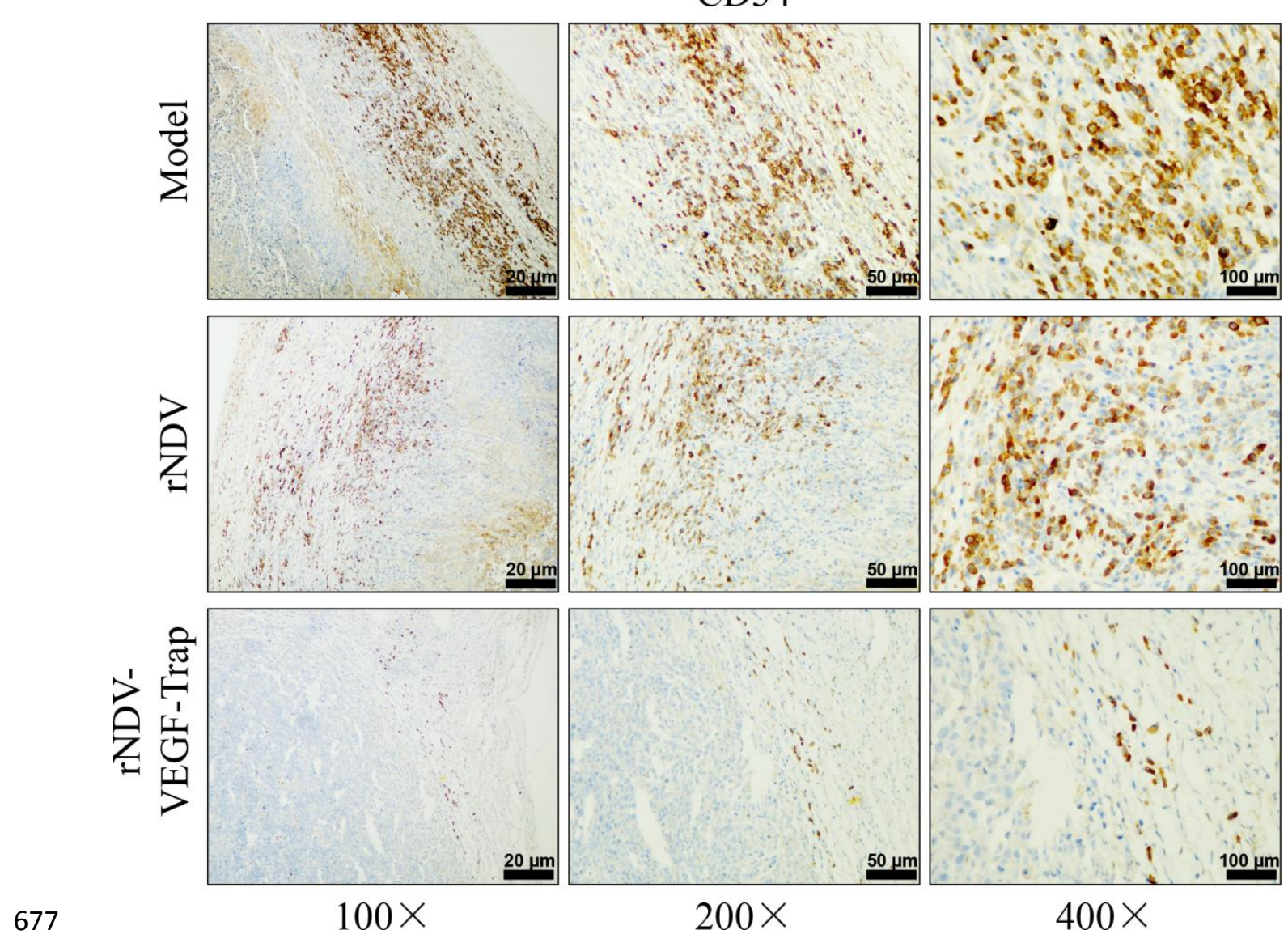
Fig.4 rNDV-VEGF-Trap suppression tumor cell viability and angiogenesis. Six-week-old female BALB/c mice were injected with $10^{6} \mathrm{CT} 26$ cells in the right groin. When the tumor size reached about $100 \mathrm{~mm}^{3}$, the mice were intratumorally injected with rNDV or rNDV-VEGF-Trap at $10^{7} \mathrm{pfu}$. The mice were euthanized and necropsy was performed after 15 days of treatment. (A) H\&E staining of CT26 tumor section after treatment. (B) CD34 in the tumor tissues were visualized by IHC assay. The result were shown in brown. The little the brown area, the less the proliferation of vascular endothelial cells was. Model: mice treated with allantoic fluid. $\mathrm{N}=3$. Magnification: 100×, 200×, 400×; scale bar $=20 \mu \mathrm{m}, 50 \mu \mathrm{m}, 100 \mu \mathrm{m}$.

A

$60 \mathrm{kDa}$

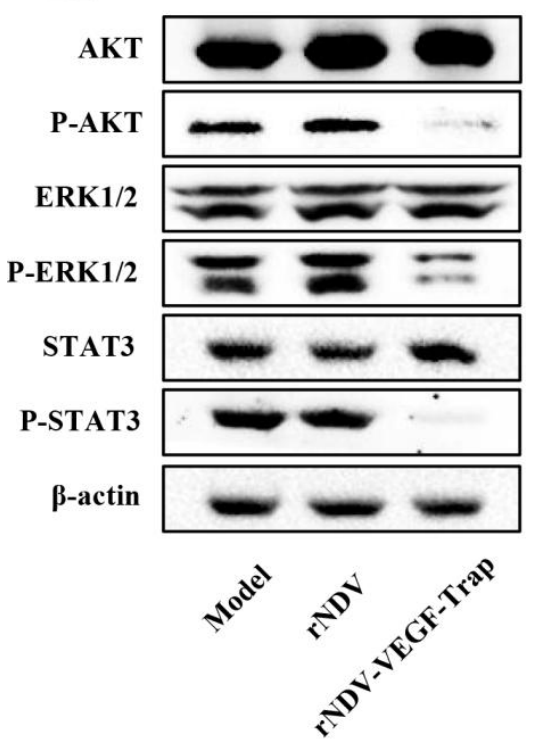

B

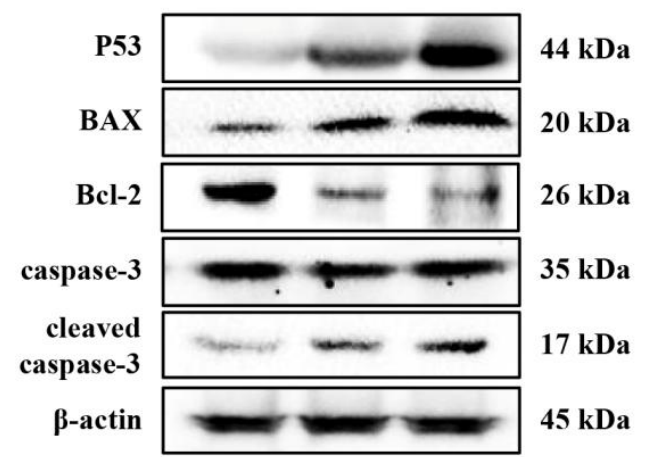

687
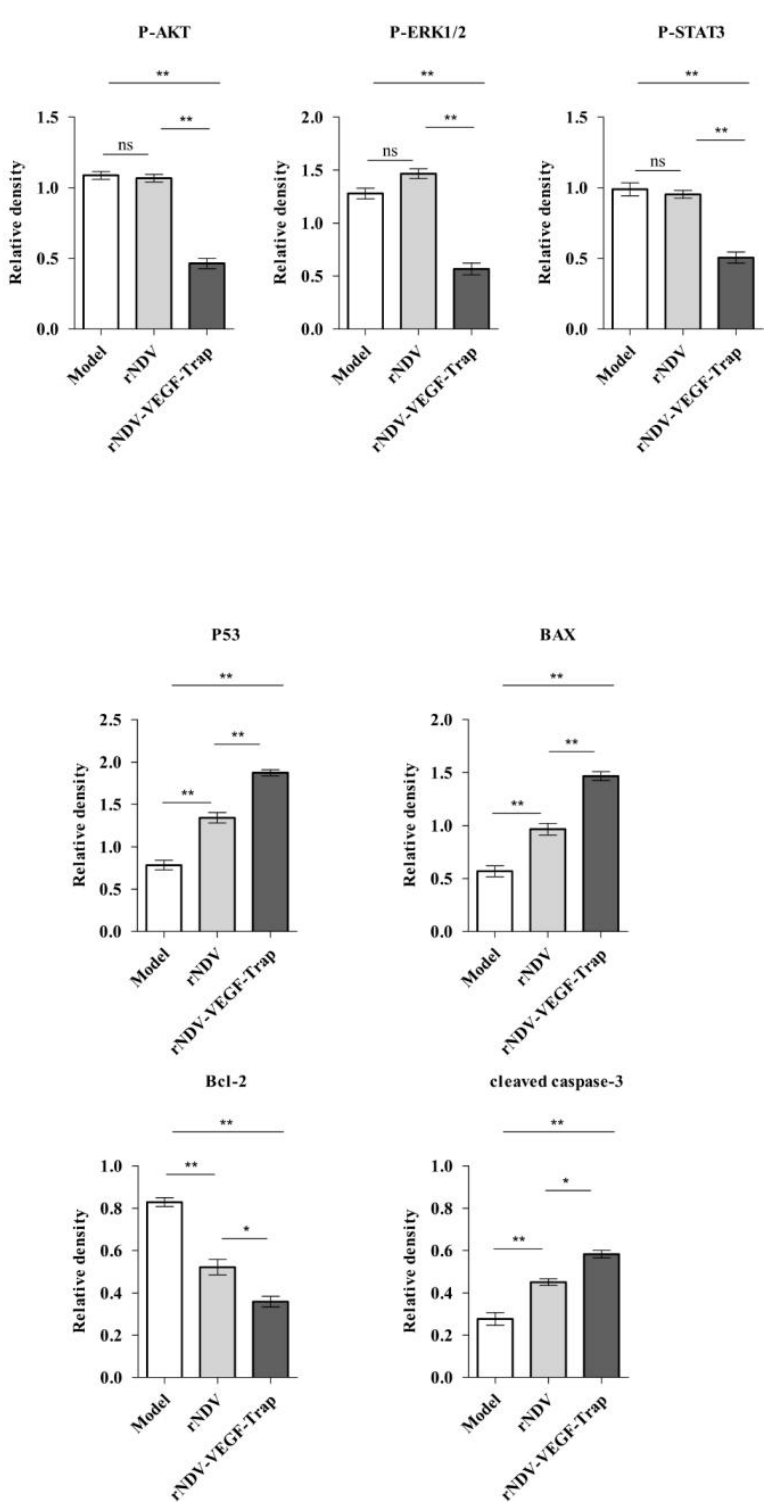

Fig.5 rNDV-VEGF-TRAP inhibits the phosphorylation of the VEGF signaling pathway related proteins AKT, ERK1/2, STAT3 and mediates the expression of the apoptosis-related proteins P53, Bcl-2, BAX, caspase-3. Six-week-old female BALB/c mice were injected with $10^{6}$ CT26 cells in the right groin. When the tumor size reached about $100 \mathrm{~mm}^{3}$, the mice were intratumorally treated with rNDV or 
rNDV-VEGF-Trap at $10^{7} \mathrm{pfu}$. The mice were euthanized and necropsy was performed after 15 days of treatment. CT26 tumor tissue (each group $0.5 \mathrm{~g}$ ) was homogenized, the supernatant was collected after centrifugation. The expression of the VEGF signaling pathway related proteins and the apoptosis-related proteins in supernatant was detected by Western blot. (A) The expression level of AKT, P-AKT, ERK1/2, P-ERK1/2, STAT3 and P-STAT3. (B) The expresssion level of P53, Bcl-2, BAX and caspase-3. $\beta$-actin was used as reference to quantify the protein bands by densitometry using ImageJ software. All data were expressed as mean $\pm \mathrm{SEM}, \mathrm{N}=3$, (ns, no significant; $* P<0.05 ; * * P<0.01)$ compared to the model.

\section{A}

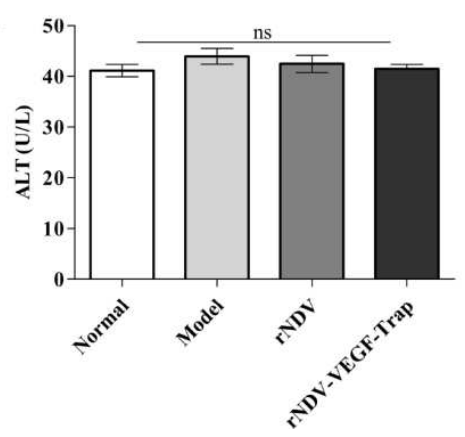

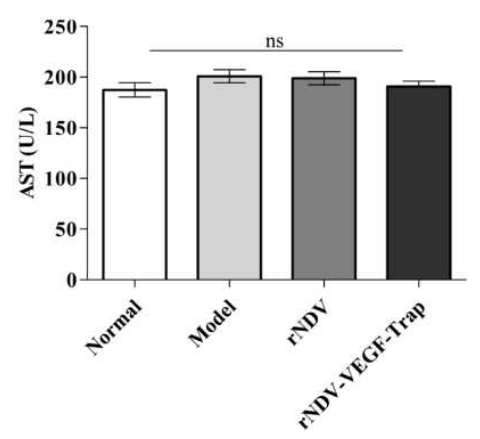

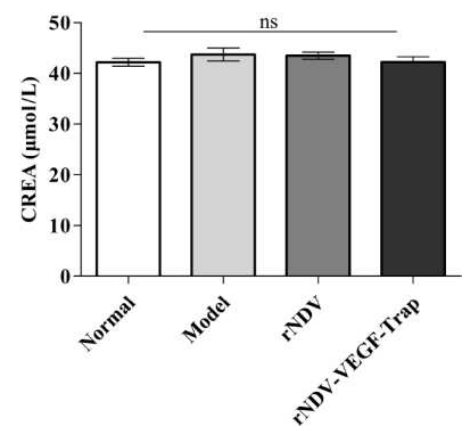

Fig. 6 rNDV-VEGF-Trap demonstrates therapeutic safety. At the end of the animal experiment, whole blood was collected through the eyeballs of the experimental mice. Then the serum was isolated from whole blood for determination of serum concentrations of AST, ALT and creatinine (A). The serum biochemistry data of the experimental mice values were expressed as mean \pm SEM. ALT, alanine aminotransferase; AST, aspartate transaminase; CREA, creatinine; Normal, normal mice. (ns, no significant). 
Figures

A

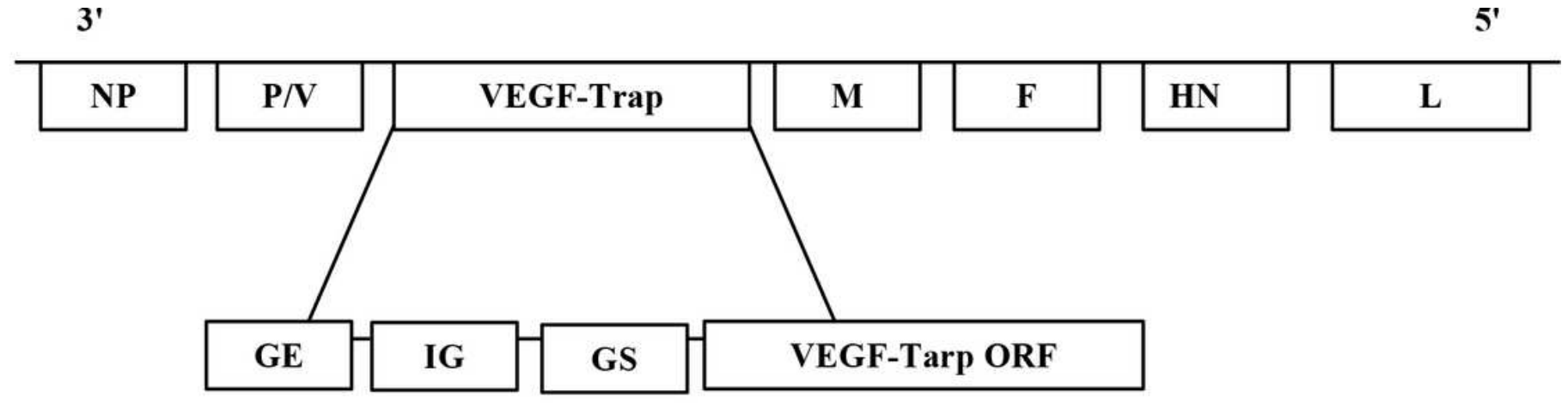

GE

IG

GS

TTAAGAAAAAA

T

ACGGGTAGAAG

B

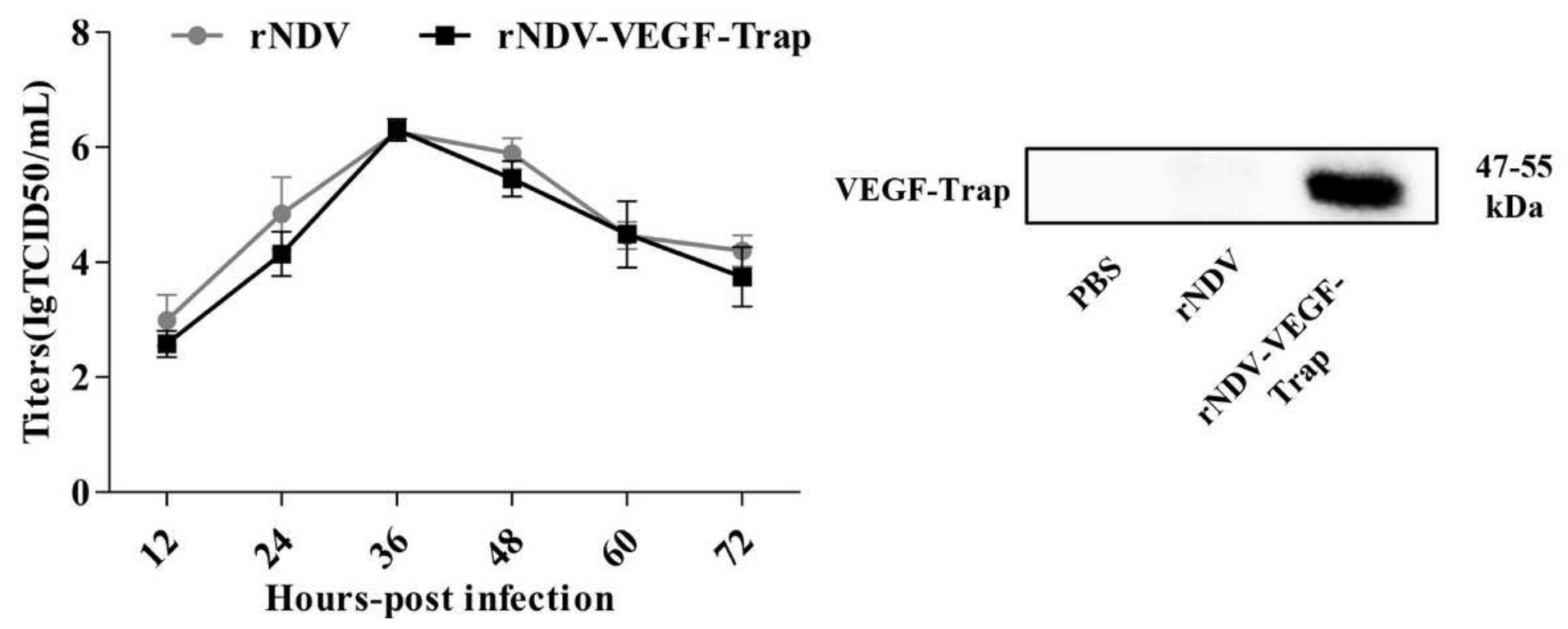

Figure 1

Schematic representation of the genomes, characterization and VEGF-Trap protein of rNDV-VEGF-Trap (A) Diagram showed insertion of the VEGF-Trap gene into the NDV genomer at the position between the $P$ and $\mathrm{M}$ genes. (B) Growth curves of rNDV-VEGF-Trap. CT26 cells were infected with rNDV and rNDV-VEGFTrap at $0.1 \mathrm{MOI}$. Cell monolayers were lysed at $12,24,48,60$ and $72 \mathrm{~h}$ post-infection for intracellular titer measurement by TCID50. (C) The expression of VEGF-Trap proteins in CT26 cells. CT26 cells were respectively infected with $1 \mathrm{MOI}$ rNDV-VEGF-Trap or rNDV and the control group received PBS. After $24 \mathrm{~h}$ incubation, cells were washed twice with cold PBS and collected by centrifugation at $500 \times \mathrm{g}$ for $5 \mathrm{~min}$ at 
$4 \bigotimes$. The cells were resuspended in lysis buffer supplemented with proteases inhibitor and after centrifugal the supernatant was used to western blot.

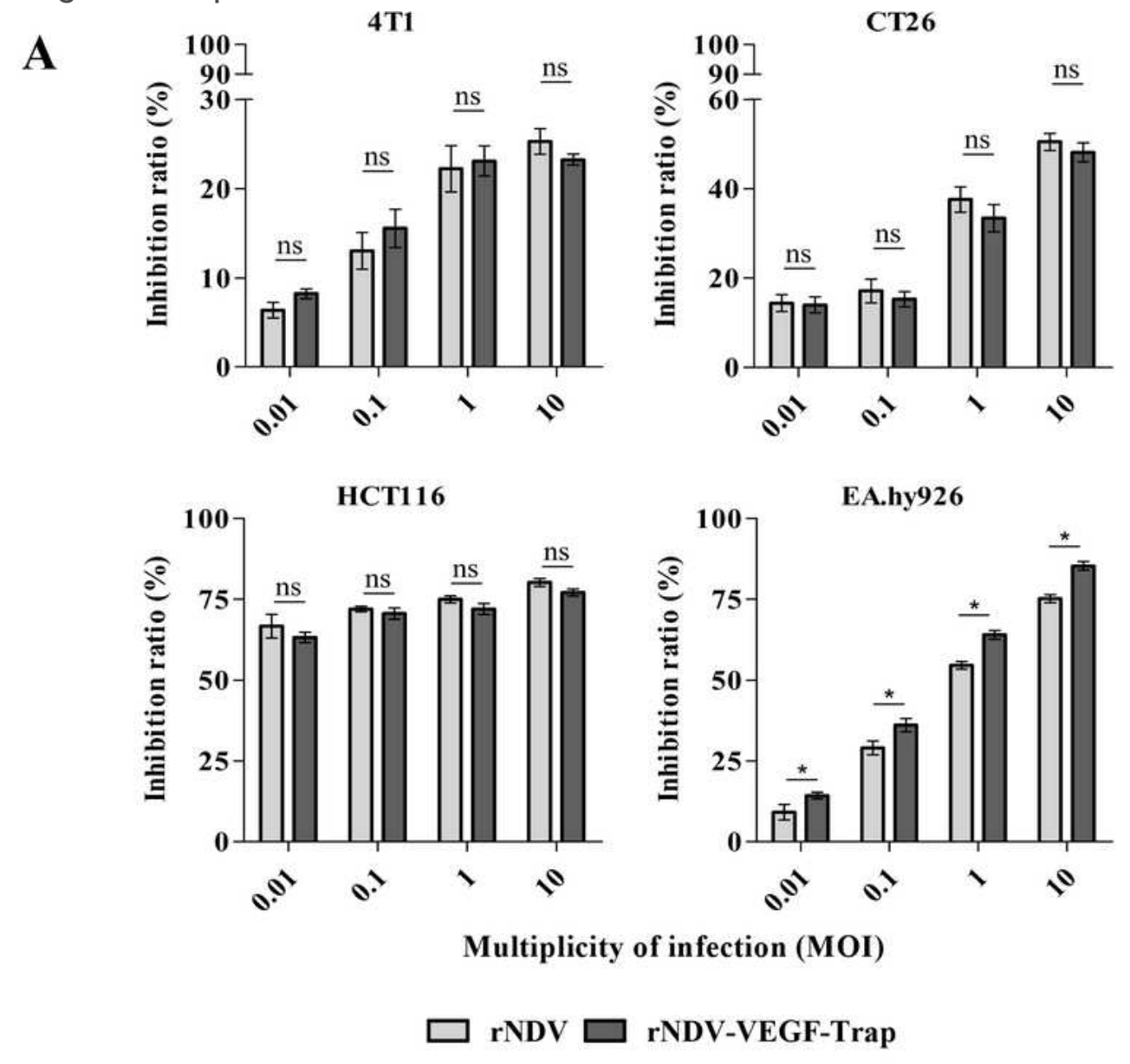

B

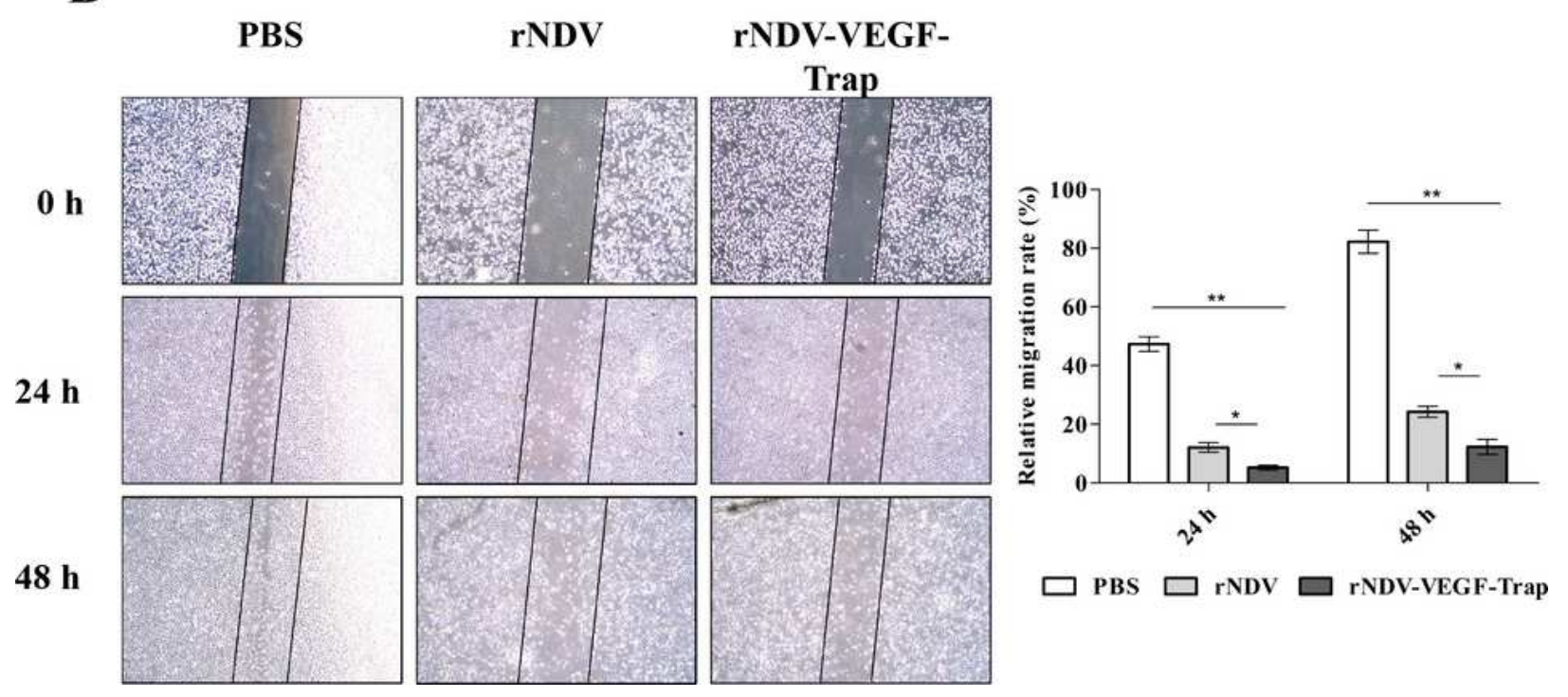

Figure 2

In vitro studies (A) Cytotoxic effects of rNDV-VEGF-Trap on cancer cells and vascular endothelial cells. 4T1, CT26, HCT116, and EA.hy926 cells incubated with PBS were used as controls. MTT method was used to measure the cell density. The data are the means \pm SEM. of triple samples $\left({ }^{\star} P<0.05 ;{ }^{\star \star} P<0.01\right)$. 
Inhibition of cell migration by recombinant virus. Wound healing assay was performed on EA.hy 926 cells, the cells were infected with $1 \mathrm{MOI}$ rNDV-VEGF-Trap or rNDV and cell migration was evaluated at regular time points post-infection. The number of migrated cells were assessed by microscope. The assay was performed in triplicate. The data are the means \pm SEM of triple samples (ns, no significant; ${ }^{*} P<0.05$; ${ }^{*} \mathrm{P}$ $<0.01)$.

A

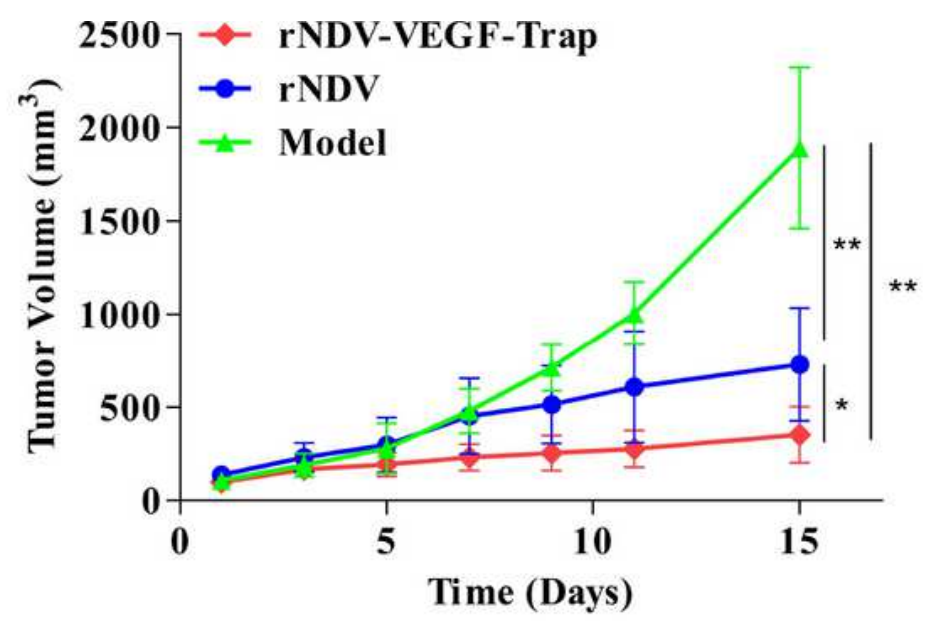

Model

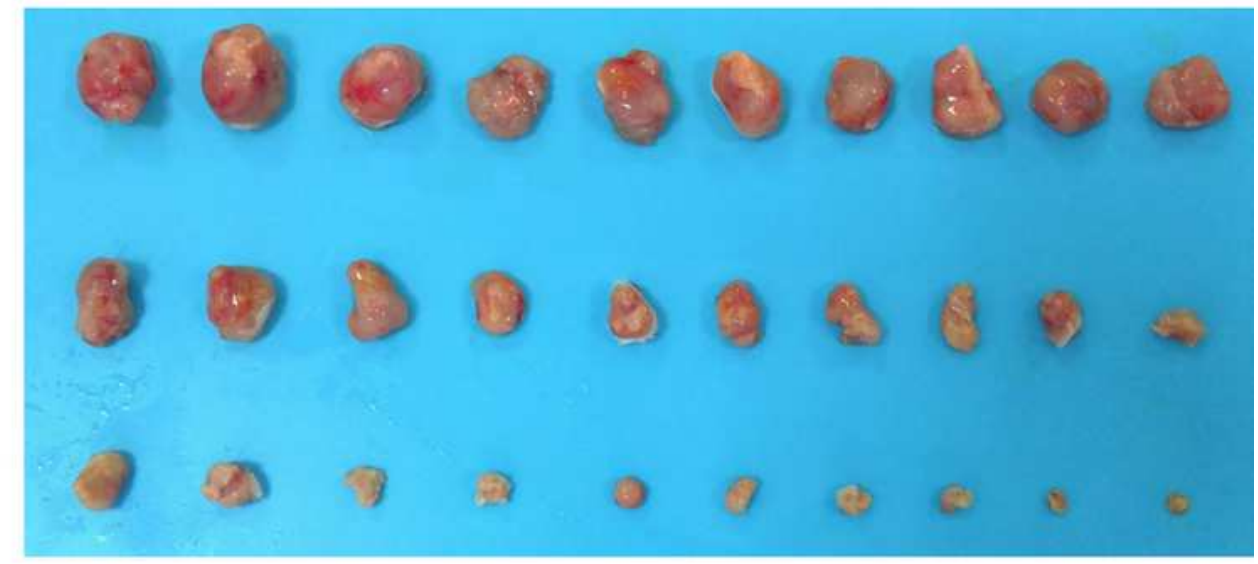

rNDVVEGF-Trap

C

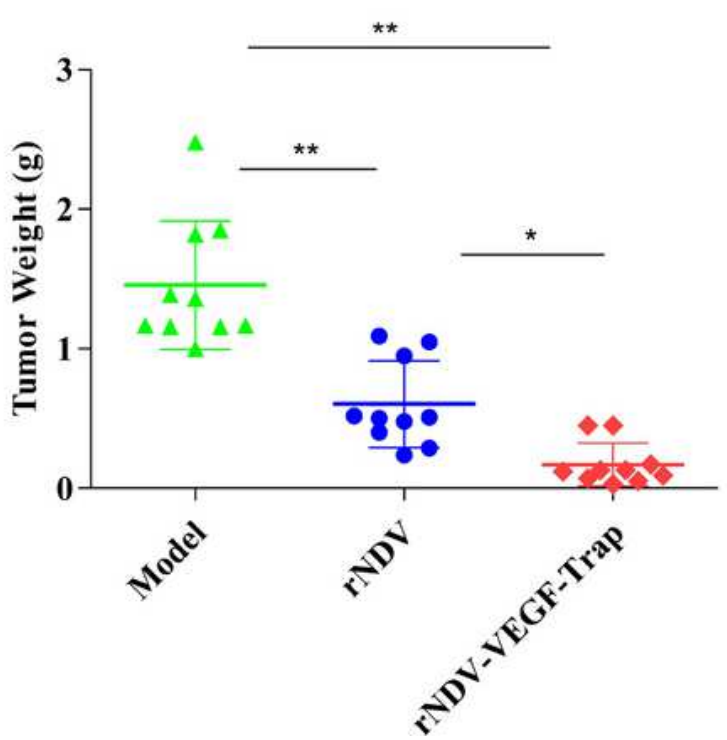

D

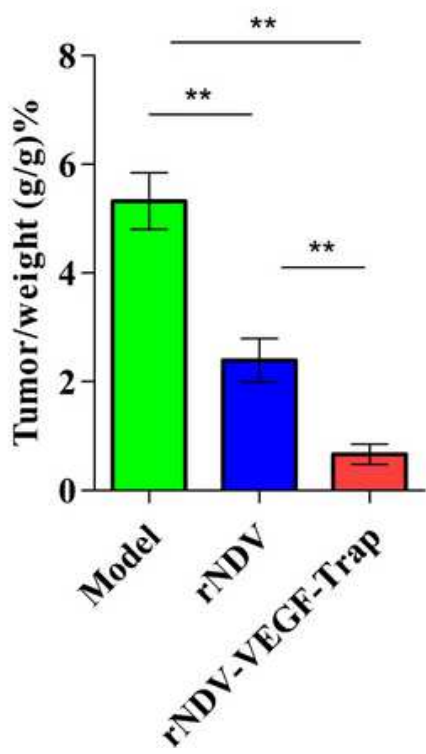

Figure 3 
rNDV-VEGF-Trap enhances inhibition of tumor growth of CT26-bearing mice. Six-week-old female BALB/c mice were injected with 106 CT26 cells in the right groin. When the tumor size reached about $100 \mathrm{~mm} 3$, the mice were intratumorally treated with rNDV or rNDV-VEGF-Trap at $107 \mathrm{pfu}$. The mice were euthanized and necropsy was performed after 15 days of treatment. (A) The tumor volume of CT26-bearing mice was measured in two dimensions every other day using digital calipers. (B) Tumor collected in the end of the experiment. (C) Tumor Weight. (D) Tumor to body weight ratio. Model: mice treated with allantoic fluid. $n=10 ; n s$, no significant; ${ }^{2} P<0.05 ; * \star P<0.01$.
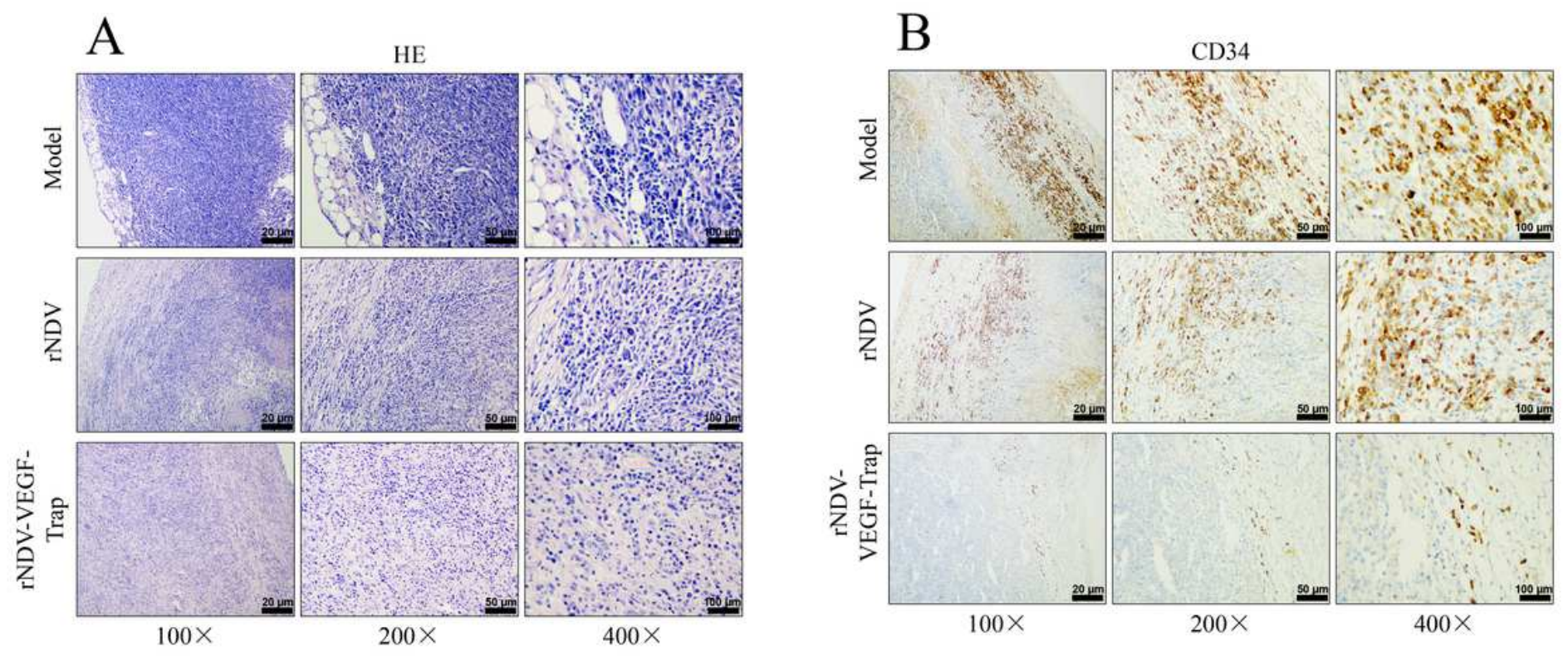

\section{Figure 4}

rNDV-VEGF-Trap suppression tumor cell viability and angiogenesis. Six-week-old female BALB/c mice were injected with 106 CT26 cells in the right groin. When the tumor size reached about $100 \mathrm{~mm} 3$, the mice were intratumorally injected with rNDV or rNDV-VEGF-Trap at $107 \mathrm{pfu}$. The mice were euthanized and necropsy was performed after 15 days of treatment. (A) H\&E staining of CT26 tumor section after treatment. (B) CD34 in the tumor tissues were visualized by IHC assay. The result were shown in brown. The little the brown area, the less the proliferation of vascular endothelial cells was. Model: mice treated with allantoic fluid. $\mathrm{N}=3$. Magnification: 100x, 200x, 400x; scale bar $=20 \mu \mathrm{m}, 50 \mu \mathrm{m}, 100 \mu \mathrm{m}$. 
A

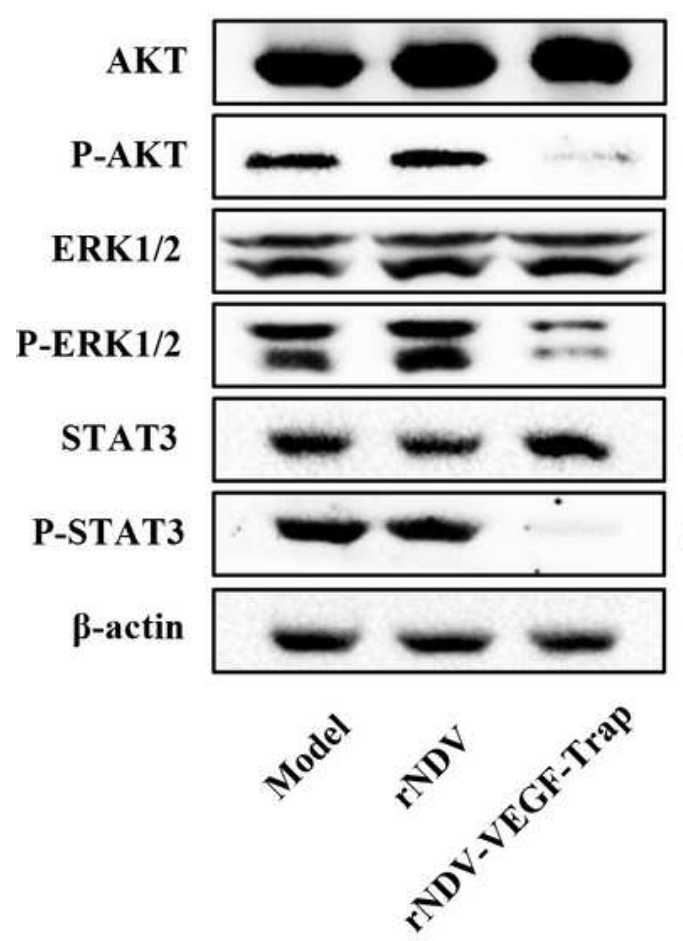

$60 \mathrm{kDa}$
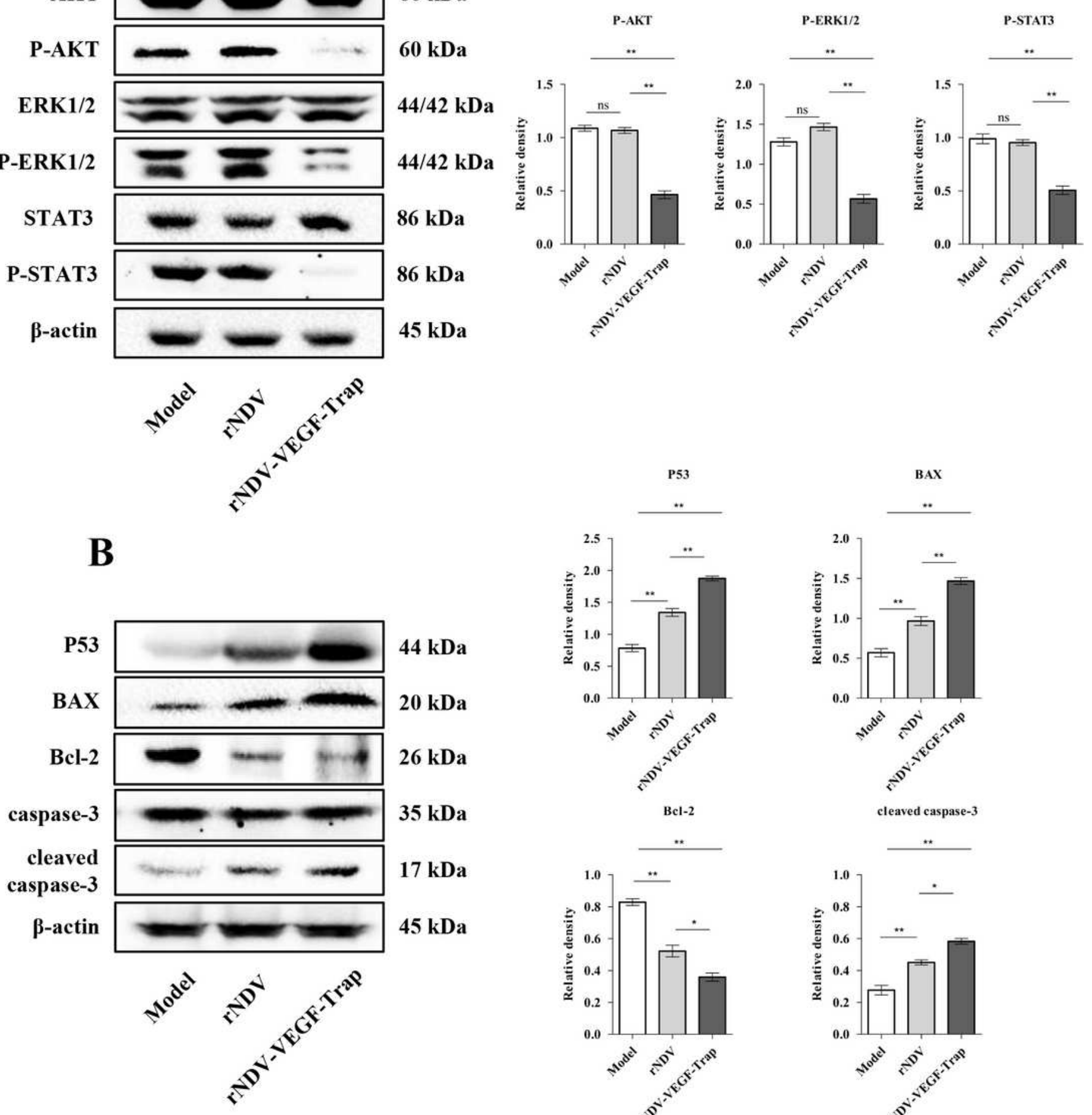

$35 \mathrm{kDa}$

17 kDa

$45 \mathrm{kDa}$
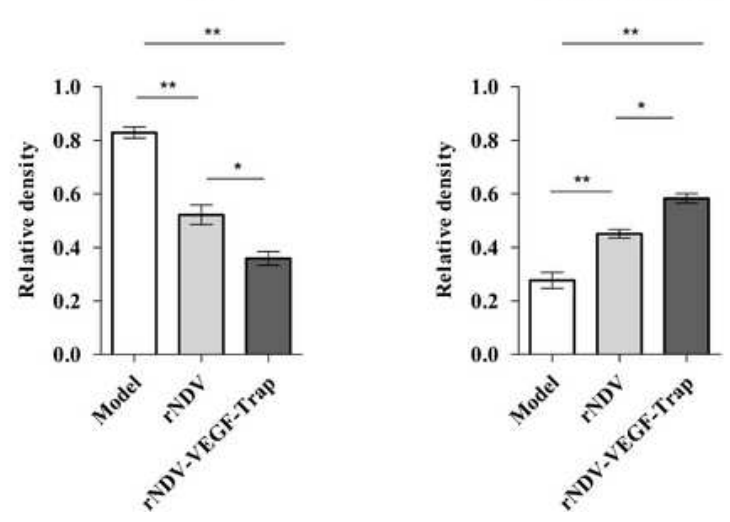

Figure 5

rNDV-VEGF-TRAP inhibits the phosphorylation of the VEGF signaling pathway related proteins AKT, ERK1/2, STAT3 and mediates the expression of the apoptosis-related proteins P53, Bcl-2, BAX, caspase-3. Six-week-old female BALB/c mice were injected with 106 CT26 cells in the right groin. When the tumor size reached about $100 \mathrm{~mm} 3$, the mice were intratumorally treated with rNDV or rNDV-VEGF-Trap at 107 pfu. The mice were euthanized and necropsy was performed after 15 days of treatment. CT26 tumor 
tissue (each group $0.5 \mathrm{~g}$ ) was homogenized, the supernatant was collected after centrifugation. The expression of the VEGF signaling pathway related proteins and the apoptosis-related proteins in supernatant was detected by Western blot. (A) The expression level of AKT, P-AKT, ERK1/2, P-ERK1/2, STAT3 and P-STAT3. (B) The expresssion level of P53, Bcl-2, BAX and caspase-3. $\beta$-actin was used as reference to quantify the protein bands by densitometry using ImageJ software. All data were expressed as mean $\pm S E M, N=3$, (ns, no significant; ${ }^{*} \mathrm{P}<0.05$; $* * \mathrm{P}<0.01$ ) compared to the model.
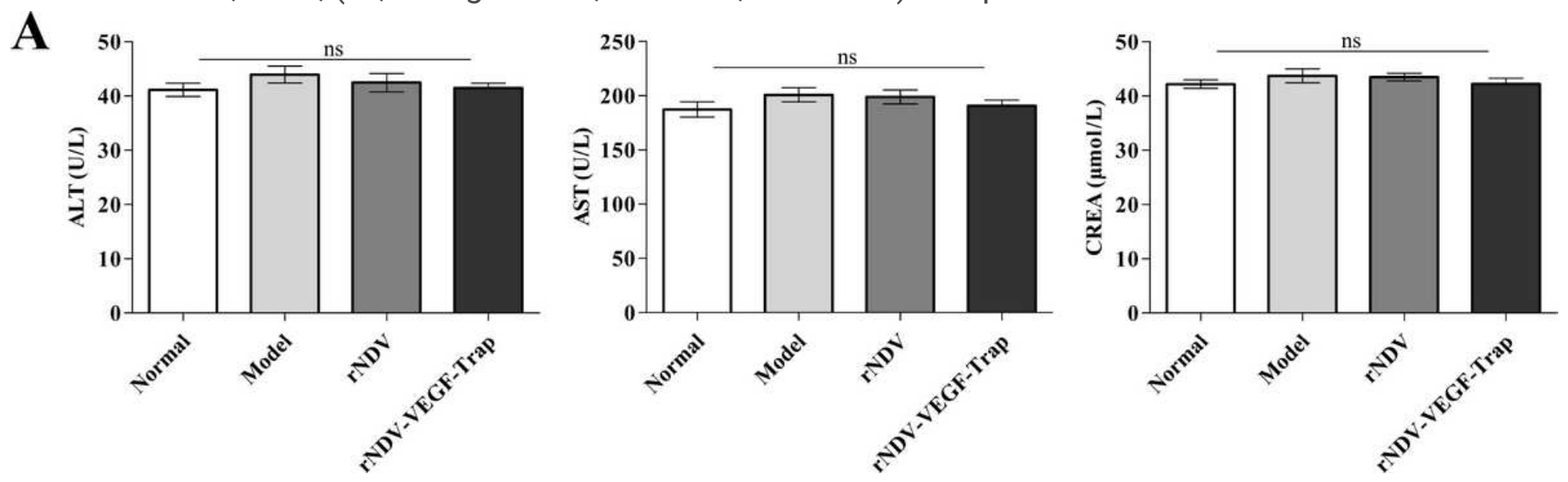

Figure 6

rNDV-VEGF-Trap demonstrates therapeutic safety. At the end of the animal experiment, whole blood was collected through the eyeballs of the experimental mice. Then the serum was isolated from whole blood for determination of serum concentrations of AST, ALT and creatinine (A). The serum biochemistry data of the experimental mice values were expressed as mean \pm SEM. ALT, alanine aminotransferase; AST, aspartate transaminase; CREA, creatinine; Normal, normal mice. (ns, no significant).

\section{Supplementary Files}

This is a list of supplementary files associated with this preprint. Click to download.

- originaldata.docx 